\title{
MULTIGRID METHODS FOR THE COMPUTATION OF SINGULAR SOLUTIONS AND STRESS INTENSITY FACTORS I: CORNER SINGULARITIES
}

\author{
SUSANNE C. BRENNER
}

\begin{abstract}
We consider the Poisson equation $-\Delta u=f$ with homogeneous Dirichlet boundary condition on a two-dimensional polygonal domain $\Omega$ with re-entrant angles. A multigrid method for the computation of singular solutions and stress intensity factors using piecewise linear functions is analyzed. When $f \in L^{2}(\Omega)$, the rate of convergence to the singular solution in the energy norm is shown to be $\mathcal{O}(h)$, and the rate of convergence to the stress intensity factors is shown to be $\mathcal{O}\left(h^{1+(\pi / \omega)-\epsilon}\right)$, where $\omega$ is the largest re-entrant angle of the domain and $\epsilon>0$ can be arbitrarily small. The cost of the algorithm is $\mathcal{O}\left(h^{-2}\right)$. When $f \in H^{1}(\Omega)$, the algorithm can be modified so that the convergence rate to the stress intensity factors is $\mathcal{O}\left(h^{2-\epsilon}\right)$. In this case the maximum error of the multigrid solution over the vertices of the triangulation is shown to be $\mathcal{O}\left(h^{2-\epsilon}\right)$.
\end{abstract}

\section{INTRODUCTION}

Let $\Omega$ be a bounded polygonal domain in $\mathbb{R}^{2}$ with at least one re-entrant angle. Consider the Poisson equation with homogeneous Dirichlet boundary condition:

$$
\begin{aligned}
-\Delta u=f & \text { in } \quad \Omega, \\
u=0 & \text { on } \quad \partial \Omega,
\end{aligned}
$$

where $f \in L^{2}(\Omega)$.

Let $\omega_{1}, \ldots, \omega_{J}$ be the internal angles of $\Omega$ which satisfy $\pi<\omega_{j}<2 \pi$ and let $p_{j}$ be the corresponding vertices. It is well known (cf. [23], [27], [32], [39]) that the unique solution $u \in H_{0}^{1}(\Omega)$ of (1.1) has the representation

$$
u=\sum_{j=1}^{J} \kappa_{j} s_{j}+w,
$$

where $w \in H^{2}(\Omega) \cap H_{0}^{1}(\Omega)$ and the singular functions $s_{j}$ are defined as follows. Let polar coordinates $\left(r_{j}, \theta_{j}\right)$ be chosen at the vertex $p_{j}$ so that the internal angle $\omega_{j}$ is spanned by the two half lines $\theta_{j}=0$ and $\theta_{j}=\omega_{j}$. Then we have

$$
s_{j}\left(r_{j}, \theta_{j}\right)=\phi_{j}\left(r_{j}\right) r_{j}^{\pi / \omega_{j}} \sin \left(\left(\pi / \omega_{j}\right) \theta_{j}\right)
$$

Received by the editor July 2, 1996.

1991 Mathematics Subject Classification. Primary 65N55, 65N30.

Key words and phrases. Multigrid, corner singularities, stress intensity factors, superconvergence.

This work was supported in part by the National Science Foundation under Grant Nos. DMS94-96275 and DMS-96-00133.

(C)1999 American Mathematical Society 
where $\phi_{j}(j=1, \ldots, J)$ are smooth cut-off functions which equal 1 identically in a neighborhood of 0 , and the supports of the $\phi_{j}$ 's are small enough so that the singular functions $s_{j}$ vanish identically on $\partial \Omega$. Moreover, the following regularity estimate holds:

$$
\|w\|_{H^{2}(\Omega)}+\sum_{j=1}^{J}\left|\kappa_{j}\right| \lesssim\|f\|_{L^{2}(\Omega)} .
$$

(Throughout this paper the statement $F \lesssim G$ means that $F$ is bounded by $G$ multiplied by a constant which is independent of the mesh size and grid level. In the statement $F \lesssim_{\epsilon} G$, the constant can depend on $\epsilon$.) The coefficient $\kappa_{j}$ can be expressed in terms of $u$ by the following extraction formula:

$$
\kappa_{j}=\frac{1}{\pi}\left\{\int_{\Omega} f s_{-j} d x+\int_{\Omega} u \Delta s_{-j} d x\right\},
$$

where the dual singular function $s_{-j}$ is defined in the polar coordinate system $\left(r_{j}, \theta_{j}\right)$ as

$$
s_{-j}\left(r_{j}, \theta_{j}\right)=\phi_{j}\left(r_{j}\right) r_{j}^{-\pi / \omega_{j}} \sin \left(\left(\pi / \omega_{j}\right) \theta_{j}\right)
$$

(cf. [6], [7], [3], [27], [24], [39]).

Formulas similar to (1.2) and (1.5) also hold for the solution of two-dimensional elasticity problems (cf. [49], [28], [29], [30], [33]). In that context, the coefficients $\kappa_{j}$ are known as stress intensity factors, and we will adopt that terminology here. These quantities play a particularly important role in linear fracture mechanics.

In view of (1.5), it is natural to compute an approximation $\kappa_{j, h}$ to $\kappa_{j}$ by

$$
\kappa_{j, h}=\frac{1}{\pi}\left\{\int_{\Omega} f s_{-j} d x+\int_{\Omega} u_{h} \Delta s_{-j} d x\right\}
$$

where $u_{h}$ is a finite element approximation of $u$. The accuracy of $\kappa_{j, h}$ computed by (1.7) depends on the accuracy of $u_{h}$, which ultimately depends on the regularity of $u$.

It is known (cf. [27]) that the singular functions $s_{j} \in H^{r}(\Omega)$ for all $r<1+\left(\pi / \omega_{j}\right)$ and $s_{j} \notin H^{1+\left(\pi / \omega_{j}\right)}(\Omega)$. It follows from (1.2) that the best one can say about the regularity of $u$ is

$$
u \in H^{r}(\Omega) \quad \forall r<1+\frac{\pi}{\omega},
$$

where

$$
\omega=\max \left(\omega_{1}, \omega_{2}, \ldots, \omega_{J}\right) .
$$

Suppose $V_{h} \subseteq H_{0}^{1}(\Omega)$ is the piecewise linear finite element space associated with a quasi-uniform triangulation $\mathcal{T}_{h}, h=\max _{T \in \mathcal{T}_{h}} \operatorname{diam} T$, and $\tilde{u}_{h} \in V_{h}$ satisfies

$$
\int_{\Omega} \nabla \tilde{u}_{h} \cdot \nabla v d x=\int_{\Omega} f v d x \quad \forall v \in V_{h} .
$$

It follows from (1.8) that for any $\epsilon>0$ we have

$$
\left|u-\tilde{u}_{h}\right|_{H^{1}(\Omega)} \lesssim \epsilon h^{(\pi / \omega)-\epsilon}\|f\|_{L^{2}(\Omega)},
$$

and

$$
\left\|u-\tilde{u}_{h}\right\|_{L^{2}(\Omega)} \lesssim \epsilon h^{2(\pi / \omega)-\epsilon}\|f\|_{L^{2}(\Omega)} .
$$


If $\tilde{\kappa}_{j, h}$ is computed by (1.7) using $\tilde{u}_{h}$, then (1.5), (1.7) and (1.12) imply that

$$
\begin{aligned}
\left|\kappa_{j}-\tilde{\kappa}_{j, h}\right| & \leq \frac{1}{\pi} \int_{\Omega}\left|\left(u-\tilde{u}_{h}\right) \Delta s_{-j}\right| d x \\
& \lesssim\left\|u-\tilde{u}_{h}\right\|_{L^{2}(\Omega)} \\
& \lesssim{ }_{\epsilon} h^{2(\pi / \omega)-\epsilon}\|f\|_{L^{2}(\Omega)} .
\end{aligned}
$$

Note that $\Delta s_{-j}=0$ near $p_{j}$, so the integration in (1.13) is only over a domain away from $p_{j}$, where $s_{-j}$ is a smooth function. Also, the estimate (1.13) cannot be improved due to the pollution effect of the corner singularity (cf. [40], [52], [53]). Thus the lack of regularity adversely affects the accuracy of the finite element solution $\tilde{u}_{h}$ and hence $\tilde{\kappa}_{j, h}$.

In this paper, we develop, on quasi-uniform grids, a multigrid method for the computation of the singular solution $u$ and the stress intensity factors $\kappa_{j}$ that obtains better estimates than (1.11)-(1.13). The basic idea is that (1.1) and (1.2) imply that $w$, the regular part of $u$, is the solution of

$$
\begin{aligned}
& -\Delta w=f+\sum_{j=1}^{J} \kappa_{j} \Delta s_{j} \quad \text { in } \quad \Omega, \\
& w=0 \quad \text { on } \quad \partial \Omega .
\end{aligned}
$$

If the $\kappa_{j}$ 's were known, we could solve (1.14) using piecewise linear finite elements, and the solution would have a good convergence rate since $w \in H^{2}(\Omega)$. Unfortunately, the $\kappa_{j}$ 's are unknown, so we instead apply the finite element method on the $k^{\text {th }}$ level to the following variational problem:

Find $\hat{w}_{k} \in H_{0}^{1}(\Omega)$ such that

$$
\int_{\Omega} \nabla \hat{w}_{k} \cdot \nabla v d x=\int_{\Omega}\left(f+\sum_{j=1}^{J} \kappa_{j, k} \Delta s_{j}\right) v d x \quad \forall v \in H_{0}^{1}(\Omega),
$$

where the approximate stress intensity factors $\kappa_{j, k}$ are computed by the extraction formula (1.5) using the approximate solution $u_{k-1}$ obtained in the $(k-1)^{\text {st }}$ level, i.e.,

$$
\kappa_{j, k}=\frac{1}{\pi}\left\{\int_{\Omega} f s_{-j} d x+\int_{\Omega} u_{k-1} \Delta s_{-j} d x\right\} .
$$

We obtain, on the $k^{\text {th }}$ level, a piecewise linear approximate solution $w_{k}$ to $\hat{w}_{k}$ by a standard multigrid algorithm. The approximate solution $u_{k}$ to $u$ is then defined to be

$$
u_{k}=\sum_{j=1}^{J} \kappa_{j, k} s_{j}+w_{k}
$$

In other words, we have a full multigrid algorithm where the right-hand side of the finite element equation changes from level to level (cf. (1.15)) and we are really computing the regular part $w$ of the solution. The improvement in the convergence rate is possible because $w$ has better regularity than $u$. We will show 
(cf. Theorem 4.1) that

$$
\begin{aligned}
\left|u-u_{k}\right|_{H^{1}(\Omega)} & \lesssim h_{k}\|f\|_{L^{2}(\Omega)}, \\
\sum_{j=1}^{J}\left|\kappa_{j}-\kappa_{j, k}\right| & \lesssim \epsilon h_{k}^{1+(\pi / \omega)-\epsilon}\|f\|_{L^{2}(\Omega)} .
\end{aligned}
$$

The estimate (1.18) is optimal for the piecewise linear finite element space, and the estimate (1.19) improves the estimate in (1.13). The computational cost of our multigrid method is proportional to the dimension of $V_{h}$, i.e., is of order $C_{\epsilon} h^{-2}$.

We also consider a second multigrid method in the case where $f \in H^{1}(\Omega)$. Let $\omega_{1}, \ldots, \omega_{N}$ be the internal angles of $\Omega$ which satisfy $\pi / 2<\omega_{j}<2 \pi$. When $f \in H^{1}(\Omega)$, the solution $u$ of (1.1) can be written as (cf. [27], [23])

$$
u=\sum_{j=1}^{N} \sum_{\ell \in \mathcal{L}_{j}} \kappa_{j, \ell} s_{j, \ell}+w
$$

where $w \in H^{3-\epsilon}(\Omega) \cap H_{0}^{1}(\Omega)$ for any $\epsilon>0$,

$$
\mathcal{L}_{j}=\left\{\ell \in \mathbb{N}: \ell\left(\pi / \omega_{j}\right)<2\right\},
$$

and in appropriate polar coordinates,

$$
s_{j, \ell}\left(r_{j}, \theta_{j}\right)=\phi_{j}\left(r_{j}\right) r_{j}^{\ell\left(\pi / \omega_{j}\right)} \sin \left(\ell\left(\pi / \omega_{j}\right) \theta_{j}\right) .
$$

Moreover, the following regularity estimates hold:

$$
\sum_{j=1}^{N} \sum_{\ell \in \mathcal{L}_{j}}\left|\kappa_{j, \ell}\right| \lesssim\|f\|_{H^{1}(\Omega)} \quad \text { and } \quad\|w\|_{H^{3-\epsilon}(\Omega)} \lesssim \epsilon\|f\|_{H^{1}(\Omega)} .
$$

The coefficient $\kappa_{j, \ell}$ can be expressed in terms of $u$ by

$$
\kappa_{j, \ell}=\frac{1}{\ell \pi}\left\{\int_{\Omega} f s_{j,-\ell} d x+\int_{\Omega} u \Delta s_{j,-\ell} d x\right\},
$$

where the dual singular function $s_{j,-\ell}$ is defined by

$$
s_{j,-\ell}\left(r_{j}, \theta_{j}\right)=\phi_{j}\left(r_{j}\right) r_{j}^{-\ell \pi / \omega_{j}} \sin \left(\ell\left(\pi / \omega_{j}\right) \theta_{j}\right) .
$$

Using similar ideas and taking advantage of a superconvergence result for linear finite elements, we develop a multigrid method that produces, at a cost of $C_{\epsilon} h_{k}^{-2}$, an approximate solution $u_{k}$ in the form

$$
u_{k}=\sum_{j=1}^{N} \sum_{\ell \in \mathcal{L}_{j}} \kappa_{j, \ell, k} s_{j, \ell}+w_{k}
$$

where $w_{k}$ is a piecewise linear function on the $k^{\text {th }}$ level triangulation, and we show that (cf. Theorem 5.4)

$$
\left|u-u_{k}\right|_{H^{1}(\Omega)} \lesssim h_{k}\|f\|_{H^{1}(\Omega)},
$$

and

$$
\sum_{j=1}^{J} \sum_{\ell \in \mathcal{L}}\left|\kappa_{j, \ell}-\kappa_{j, \ell, k}\right| \lesssim_{\epsilon} h_{k}^{2-\epsilon}\|f\|_{H^{1}(\Omega)}
$$


As a corollary (cf. Corollary 5.6), we obtain

$$
\max _{p}\left|u(p)-u_{k}(p)\right| \lesssim_{\epsilon} h_{k}^{2-\epsilon}\|f\|_{H^{1}(\Omega)},
$$

where the maximum is taken over all the vertices $p$ of the $k^{\text {th }}$ level triangulation.

Corner singularities can also be overcome by the method of local grid refinement (cf. [1], [2]). Using this method, the number of degrees of freedom is of order $\mathcal{O}\left(h^{-2}\right)$ and the error of the computed stress intensity factor is of order $\mathcal{O}\left(h^{2}\right)$. This method also has the advantage that it does not require the knowledge of the exact forms of the singular functions. However, it is more difficult in this approach to use multilevel techniques (cf. [57], [58], [59], [60], [45]).

Another method for the computation of the stress intensity factors is the dual singular function method (DSFM) (cf. [25], [7], [8]) where extraction formulas are also used. In each iterative step of the DSFM, a finite element equation of the form (1.15) is solved on the same grid. The algorithms developed in this paper can in some sense be viewed as a multigrid version of the DSFM.

We should also mention that in [38] a multigrid method based on an extrapolation technique is proposed, where $\mathcal{O}\left(h^{2-\epsilon}\right)$ convergence for the stress intensity factors with cost $\mathcal{O}\left(h^{-2}\right)$ is claimed. However, the result in [38] is obtained under the unproven assumption that the standard multigrid method using piecewise linear finite elements is a contraction with respect to the $L^{2}$ norm in the presence of corner singularities. There are also no numerical results in [38] to corroborate the claim.

The rest of this paper is organized as follows. The multigrid algorithms are defined in Section 2 and the convergence analyses are carried out in Sections 3, 4, and 5. The results of numerical experiments are presented in Section 6. Section 7 contains some concluding remarks.

\section{The MUltigrid ALGORIthms}

Let $\left\{\mathcal{T}_{k}\right\}, k \geq 1$, be a family of triangulations of $\Omega$, where $\mathcal{T}_{k+1}$ is obtained from $\mathcal{T}_{k}$ by regular subdivision, i.e., by connecting the midpoints of the edges of the triangles in $\mathcal{T}_{k}$, and define $h_{k}=\max _{T \in \mathcal{T}_{k}} \operatorname{diam} T$. Let $V_{k}=\left\{v \in H_{0}^{1}(\Omega):\left.v\right|_{T} \in\right.$ $\left.\mathcal{P}_{1}(T) \quad \forall T \in \mathcal{T}_{k}\right\}$ be the piecewise linear finite element space associated with $\mathcal{T}_{k}$.

We define the discrete inner product $(\cdot, \cdot)_{k}$ by

$$
\left(v_{1}, v_{2}\right)_{k}=h_{k}^{2} \sum v_{1}(p) v_{2}(p) \quad \forall v_{1}, v_{2} \in V_{k},
$$

where the summation is taken over all the vertices $p$ of $\mathcal{T}_{k}$. The operators $A_{k}$ : $V_{k} \longrightarrow V_{k}$ and $I_{k}^{k-1}: V_{k} \longrightarrow V_{k-1}$ are defined by

$$
\left(A_{k} v_{1}, v_{2}\right)_{k}=\int_{\Omega} \nabla v_{1} \cdot \nabla v_{2} d x \quad \forall v_{1}, v_{2} \in V_{k}
$$

and

$$
\left(I_{k}^{k-1} v, w\right)_{k-1}=(v, w)_{k} \quad \forall v \in V_{k}, w \in V_{k-1}\left(\subseteq V_{k}\right) .
$$

Clearly, $A_{k}$ is symmetric positive definite with respect to $(\cdot, \cdot)_{k}$. Since $(v, v)_{k}$ is equivalent to $\|v\|_{L^{2}(\Omega)}$ for $v \in V_{k}$, it follows from (2.2) and a standard inverse estimate (cf. [20], [16]) that the spectral radius $\rho\left(A_{k}\right)$ satisfies $\rho\left(A_{k}\right) \lesssim h_{k}^{-2}$. 
For the convergence analysis in Section 3, we also need the Ritz projection operator $P_{k}: H_{0}^{1}(\Omega) \longrightarrow V_{k}$ defined by

$$
\int_{\Omega} \nabla\left(\zeta-P_{k} \zeta\right) \cdot \nabla v d x=0 \quad \forall \zeta \in H_{0}^{1}(\Omega), v \in V_{k}
$$

It is easy to see that

$$
I_{k}^{k-1} A_{k}=A_{k-1} P_{k-1}
$$

The following is the standard one-sided $k^{\text {th }}$-level multigrid iteration: For $p=1$, this is the usual $V(m, 0)$ cycle, and for $p=2$, this is the usual $W(m, 0)$ cycle.

The $\mathbf{k}^{\text {th }}$ level iteration. The $k^{\text {th }}$ level iteration with initial guess $z_{0}$ yields $M G\left(k, z_{0}, g\right)$ as an approximate solution to the equation

$$
A_{k} z=g .
$$

For $k=1, M G\left(1, z_{0}, g\right)$ is the solution obtained from an exact solver. In other words,

$$
M G\left(1, z_{0}, g\right)=A_{1}^{-1} g
$$

For $k>1$, there are two steps.

Smoothing Step Let $z_{l} \in V_{k}(1 \leq l \leq m)$ be defined recursively by the equations

$$
z_{l}=z_{l-1}+\frac{1}{\Lambda_{k}}\left(g-A_{k} z_{l-1}\right), \quad 1 \leq l \leq m
$$

where $m$ is a positive integer independent of $k$, and $\Lambda_{k}:=C h_{k}^{-2}$ dominates the spectral radius of $A_{k}$.

Correction Step Let $\bar{g}=I_{k}^{k-1}\left(g-A_{k} z_{m}\right)$ and $q_{i} \in V_{k-1}(0 \leq i \leq p, p=1$ or 2$)$ be defined recursively by

$$
q_{0}=0 \quad \text { and } \quad q_{i}=M G\left(k-1, q_{i-1}, \bar{g}\right), 1 \leq i \leq p .
$$

The output is obtained by combining the two steps:

$$
M G\left(k, z_{0}, g\right)=z_{m}+q_{p}
$$

Remark. We use Richardson relaxation in (2.8) to simplify the analysis in Section 3. Other smoothers (cf. [37], [31], [4], [36], [10], [12]) can of course be used.

Full multigrid algorithm I. When $f \in L^{2}(\Omega)$, we use a nested iteration (where each $k^{\text {th }}$ level iteration is applied $n$ times $)$ to compute $\kappa_{j, k} \in \mathbb{R}(1 \leq j \leq J)$ and $w_{k} \in V_{k}$ so that $\kappa_{j, k}$ is a good approximation of $\kappa_{j}$, and $u_{k}=\sum_{j=1}^{J} \kappa_{j, k} s_{j}+w_{k}$ is a good approximation of the solution $u$ of (1.1) (cf. (1.2)).

The nested iteration. For $k=1$,

$$
w_{1}=A_{1}^{-1} g_{1},
$$

where $g_{1} \in V_{1}$ is defined by

$$
\left(g_{1}, v\right)_{1}=\int_{\Omega} f v d x \quad \forall v \in V_{1},
$$

and we set

$$
\begin{aligned}
\kappa_{j, 1} & =0 \quad \text { for } \quad 1 \leq j \leq J, \\
u_{1} & =w_{1} .
\end{aligned}
$$


For $k \geq 2$, the numbers $\kappa_{j, k} \in \mathbb{R}$ are computed by

$$
\kappa_{j, k}=\frac{1}{\pi}\left\{\int_{\Omega} f s_{-j} d x+\int_{\Omega} u_{k-1} \Delta s_{-j} d x\right\} \quad \text { for } 1 \leq j \leq J,
$$

and $w_{k} \in V_{k}$ is obtained recursively by

$$
\begin{aligned}
w_{k, 0} & =w_{k-1}, \\
w_{k, l} & =M G\left(k, w_{k, l-1}, g_{k}\right) \quad \text { for } 1 \leq l \leq n, \\
w_{k} & =w_{k, n},
\end{aligned}
$$

where $n$ is a positive integer independent of $k$, and $g_{k} \in V_{k}$ is defined by

$$
\left(g_{k}, v\right)_{k}=\int_{\Omega}\left(f+\sum_{j=1}^{J} \kappa_{j, k} \Delta s_{j}\right) v d x \quad \forall v \in V_{k}
$$

We then define $u_{k}$ by

$$
u_{k}=\sum_{j=1}^{J} \kappa_{j, k} s_{j}+w_{k}
$$

Full multigrid algorithm II. When $f \in H^{1}(\Omega)$, we use a nested iteration (where each $k^{\text {th }}$ level iteration is applied $n$ times) to compute $\kappa_{j, \ell, k} \in \mathbb{R}(1 \leq$ $j \leq N, \ell \in \mathcal{L}_{j}$ ) and $w_{k} \in V_{k}$ so that $\kappa_{j, \ell, k}$ is a good approximation of $\kappa_{j, \ell}$, and $u_{k}=\sum_{j=1}^{N} \sum_{\ell \in \mathcal{L}_{j}} \kappa_{j, \ell, k} s_{j, \ell}+w_{k}$ is a good approximation of the solution $u$ of (1.1) (cf. $(1.20))$.

The nested iteration For $k=1$,

$$
w_{1}=A_{1}^{-1} g_{1},
$$

where $g_{1} \in V_{1}$ is defined by

$$
\left(g_{1}, v\right)_{1}=\int_{\Omega} f v d x \quad \forall v \in V_{1},
$$

and we set

$$
\begin{aligned}
\kappa_{j, \ell, 1} & =0 \quad \text { for } \quad 1 \leq j \leq N, \ell \in \mathcal{L}_{j}, \\
u_{1} & =w_{1} .
\end{aligned}
$$

For $k \geq 2$, the numbers $\kappa_{j, \ell, k} \in \mathbb{R}$ are computed by

$$
\kappa_{j, \ell, k}=\frac{1}{\ell \pi}\left\{\int_{\Omega} f s_{j,-\ell} d x+\int_{\Omega} u_{k-1} \Delta s_{j,-\ell} d x\right\} \quad \text { for } 1 \leq j \leq N, \ell \in \mathcal{L}_{j}
$$

and $w_{k} \in V_{k}$ is obtained recursively by

$$
\begin{aligned}
w_{k, 0} & =I_{k-1}^{k} w_{k-1}, \\
w_{k, l} & =M G\left(k, w_{k, l-1}, g_{k}\right) \quad \text { for } 1 \leq l \leq n, \\
w_{k} & =w_{k, n},
\end{aligned}
$$

where $n$ is a positive integer independent of $k$, and $g_{k} \in V_{k}$ is defined by

$$
\left(g_{k}, v\right)_{k}=\int_{\Omega}\left(f+\sum_{j=1}^{N} \sum_{\ell \in \mathcal{L}_{j}} \kappa_{j, \ell, k} \Delta s_{j, \ell}\right) v d x \quad \forall v \in V_{k} .
$$


We then define $u_{k}$ by

$$
u_{k}=\sum_{j=1}^{N} \sum_{\ell \in \mathcal{L}_{j}} \kappa_{j, \ell, k} s_{j, \ell}+w_{k} .
$$

The intergrid transfer operator $I_{k-1}^{k}: V_{k-1} \longrightarrow V_{k}$ in (2.24) is defined as follows. We assume that $\mathcal{T}_{1}$ is also obtained by connecting the midpoints of a coarser triangulation $\mathcal{T}_{0}$. Let $Q_{k} \subset H_{0}^{1}(\Omega)(k=0,1,2, \ldots)$ be the quadratic Lagrange finite element space associated with $\mathcal{T}_{k}$. Let $\mathcal{I}_{k-2}^{k-1}: Q_{k-2} \longrightarrow V_{k-1}$ and $\mathcal{I}_{k-2}^{k}: Q_{k-2} \longrightarrow V_{k}$ be the nodal interpolation operators. Since $Q_{k-2}$ and $V_{k-1}$ share the same nodal points, it is clear that $\mathcal{I}_{k-2}^{k-1}$ is an isomorphism from $Q_{k-2}$ onto $V_{k-1}$. We define, for $k=2,3, \ldots$,

$$
I_{k-1}^{k}=\mathcal{I}_{k-2}^{k} \circ\left(\mathcal{I}_{k-2}^{k-1}\right)^{-1} .
$$

This more sophisticated intergrid transfer operator is needed to maintain a higher convergence rate for this full multigrid algorithm.

Remark. A standard argument (cf. [5]) shows that the costs for both full multigrid algorithms are proportional to $\operatorname{dim} V_{k}$.

\section{Contraction PROperties OF THE $k^{\text {TH }}$ LEVEL ITERATION}

We have the following result (cf. [9], [10], [13], [14]) for the convergence of the $k^{\text {th }}$ level iteration in the energy norm.

Lemma 3.1. Let $p=1$ ( $V$-cycle) or 2 ( $W$-cycle) and $m \geq 1$ in the $k^{\text {th }}$ level iteration. Then there exists a $\delta<1$, independent of $k$, such that

$$
\left|z-M G\left(k, z_{0}, g\right)\right|_{H^{1}(\Omega)} \leq \delta\left|z-z_{0}\right|_{H^{1}(\Omega)} .
$$

Remark. The estimate (3.1) was obtained in [13] and [14] for the symmetric $V$ cycle algorithm. Since the finite element spaces $V_{k}$ are conforming and nested, it also holds (cf. [37]) for the one-sided $V$-cycle and $W$-cycle algorithms described in Section 2. Moreover, the contraction number $\delta$ for the $W$-cycle algorithm can be made arbitrarily small by increasing the number of smoothing steps $m$ (cf. [5]).

We also have the following convergence result for the $k^{\text {th }}$ level iteration in the $\|\cdot\|_{H^{1-(\pi / \omega)+\epsilon(\Omega)}}$ norm.

Lemma 3.2. Let $p=2(W$-cycle $), 0<\delta<1,0<\epsilon<\pi / \omega$ and $\alpha_{\epsilon}=1-(\pi / \omega)+\epsilon \neq$ $1 / 2$. If the number of smoothing steps $m$ in the $k^{\text {th }}$ level iteration is sufficiently large, then we have

$$
\left\|z-M G\left(k, z_{0}, g\right)\right\|_{H^{\alpha_{\epsilon}(\Omega)}} \leq \delta\left\|z-z_{0}\right\|_{H^{\alpha_{\epsilon}(\Omega)}} .
$$

Proof. We follow the methodology in [5]. First we consider the two-grid algorithm where $q_{p}$ in (2.10) is replaced by (cf. (2.5))

$$
q=A_{k-1}^{-1} \bar{g}=A_{k-1}^{-1} I_{k}^{k-1} A_{k}\left(z-z_{m}\right)=P_{k-1}\left(z-z_{m}\right) .
$$

Let $R_{k}: V_{k} \longrightarrow V_{k}$ be defined by

$$
R_{k}=I-\frac{1}{\Lambda_{k}} A_{k} .
$$


Then, by (2.8) and (3.4), we have

$$
z-z_{m}=R_{k}^{m}\left(z-z_{0}\right) .
$$

It follows from (3.3) and (3.5) that the error of the output $\tilde{z}=z_{m}+q$ of the two-grid method is given by

$$
z-\tilde{z}=z-z_{m}-q=\left(I-P_{k-1}\right)\left(z-z_{m}\right)=\left(I-P_{k-1}\right) R_{k}^{m}\left(z-z_{0}\right) .
$$

For $0 \leq s \leq 1$, we define

$$
\|v\|_{s}=\sqrt{\left(A_{k}^{s} v, v\right)_{k}} \quad \forall v \in V_{k}
$$

We immediately have

$$
\|v\|_{1} \leq\left[\rho\left(A_{k}^{1-s}\right)\right]^{1 / 2}\|v\|_{s} \lesssim h_{k}^{s-1}\|v\|_{s} \quad \forall v \in V_{k}, 0 \leq s \leq 1,
$$

and since $\Lambda_{k}=C h_{k}^{-2}$ dominates the spectral radius of $A_{k}$,

$$
\left\|R_{k} v\right\|_{s} \leq\|v\|_{s} \quad \forall v \in V_{k}, 0 \leq s \leq 1 .
$$

The effect of the smoothing step is measured by the following smoothing property:

$$
\begin{aligned}
\left\|R_{k}^{m} v\right\|_{1} & =\left(\left(A_{k} R_{k}^{m} v, R_{k}^{m} v\right)_{k}\right)^{1 / 2} \\
& \leq \Lambda_{k}^{s / 2}\left[\rho\left(\Lambda_{k}^{-s} A_{k}^{s} R_{k}^{2 m}\right)\right]^{1 / 2}\left(A_{k}^{1-s} v, v\right)_{k}^{1 / 2} \\
& \lesssim h_{k}^{-s}\left[\sup _{0 \leq t \leq 1} t^{s}(1-t)^{2 m}\right]^{1 / 2}\|v\|_{1-s} \\
& \lesssim h_{k}^{-s} m^{-s / 2}\|v\|_{1-s} \quad \forall v \in V_{k}, 0 \leq s \leq 1 .
\end{aligned}
$$

The effect of the correction step is given by the following approximation property:

$$
\left\|v-P_{k-1} v\right\|_{H^{\alpha_{\epsilon}(\Omega)}} \lesssim_{\epsilon} h_{k}^{\pi / \omega-\epsilon}\left|v-P_{k-1} v\right|_{H^{1}(\Omega)} \quad \forall v \in H_{0}^{1}(\Omega) .
$$

We will establish (3.11) by a duality argument. Let $\phi \in H^{-\alpha_{\epsilon}}(\Omega)$ and $\zeta \in H_{0}^{1}(\Omega)$ satisfy

$$
\int_{\Omega} \nabla \zeta \cdot \nabla v d x=\phi(v) \quad \forall v \in H_{0}^{1}(\Omega) .
$$

It is well known (cf. [23]) that $\zeta \in H^{1+(\pi / \omega)-\epsilon}(\Omega)$ and

$$
\|\zeta\|_{H^{1+(\pi / \omega)-\epsilon(\Omega)}} \lesssim \epsilon\|\phi\|_{H^{-\alpha \epsilon}(\Omega)} .
$$

Let $\Pi_{k}$ be the nodal interpolation operator associated with $V_{k}$. It follows from standard interpolation error estimates (cf. [20], [16]) and (3.13) that

$$
\left|\zeta-\Pi_{k} \zeta\right|_{H^{1}(\Omega)} \lesssim_{\epsilon} h_{k}^{(\pi / \omega)-\epsilon}\|\phi\|_{H^{-\alpha}(\Omega)} .
$$

Using (2.4), (3.12) and (3.14), we have

$$
\begin{aligned}
\phi\left(v-P_{k-1} v\right) & =\int_{\Omega} \nabla \zeta \cdot \nabla\left(v-P_{k-1} v\right) d x \\
& =\int_{\Omega} \nabla\left(\zeta-\Pi_{k-1} \zeta\right) \cdot \nabla\left(v-P_{k-1} v\right) d x \\
& \lesssim_{\epsilon}^{(\pi / \omega)-\epsilon}\|\phi\|_{H^{-\alpha_{\epsilon}(\Omega)}}\left|v-P_{k-1} v\right|_{H^{1}(\Omega)} .
\end{aligned}
$$

The estimate (3.11) follows from (3.15) and the duality formula

$$
\|\eta\|_{H^{\alpha_{\epsilon}(\Omega)}}=\sup \left[\phi(\eta) /\|\phi\|_{H^{-\alpha_{\epsilon}(\Omega)}}\right] \quad \forall \eta \in H_{0}^{\alpha_{\epsilon}}(\Omega),
$$

where the supremum is taken over all $\phi \in H^{-\alpha_{\epsilon}}(\Omega) \backslash\{0\}$. 
The final ingredient is the relation between the mesh dependent norm $\|\cdot\|_{s}$ and the Sobolev norm $\|\cdot\|_{H^{s}(\Omega)}$ on $V_{k}$. First of all, we have $|v|_{H^{1}(\Omega)}=\|v\|_{1}$ and $\|v\|_{L^{2}(\Omega)} \lesssim\|v\|_{0}$ for all $v \in V_{k}$. Interpolating these estimates (cf. [50]), we have

$$
\|v\|_{H^{s}(\Omega)} \lesssim\|v\|_{s} \quad \forall v \in V_{k}, 0 \leq s \leq 1 .
$$

On the other hand, there exists (cf. [21], [48]) an interpolation operator $\pi_{k}$ : $L^{2}(\Omega) \longrightarrow V_{k}$ such that

$$
\begin{aligned}
\left\|\pi_{k} v\right\|_{0} \lesssim\left\|\pi_{k} v\right\|_{L^{2}(\Omega)} \lesssim\|v\|_{L^{2}(\Omega)} & & \forall v \in L^{2}(\Omega), \\
\left\|\pi_{k} v\right\|_{1} \lesssim\left\|\pi_{k} v\right\|_{H^{1}(\Omega)} \lesssim\|v\|_{H^{1}(\Omega)} & & \forall v \in H_{0}^{1}(\Omega), \\
\pi_{k} v=v & & \forall v \in V_{k} .
\end{aligned}
$$

For $0<s<1$ and $s \neq 1 / 2$, we can interpolate (3.18) and (3.19) (cf. [50]) to obtain

$$
\left\|\pi_{k} v\right\|_{s} \lesssim\|v\|_{H^{s}(\Omega)} \quad \forall v \in H_{0}^{s}(\Omega) .
$$

Combining (3.20) and (3.21), we find

$$
\|v\|_{s} \lesssim\|v\|_{H^{s}(\Omega)} \quad \forall v \in V_{k}, 0 \leq s \leq 1, s \neq 1 / 2 .
$$

In other words, the mesh dependent norm $\|\cdot\|_{s}$ and the Sobolev norm $\|\cdot\|_{H^{s}(\Omega)}$ are equivalent on $V_{k}$ as long as $s \neq 1 / 2$.

Therefore, from (3.6)-(3.11) and (3.22), we have the following error estimate for the two-grid algorithm:

$$
\begin{aligned}
\|z-\tilde{z}\|_{H^{\alpha_{\epsilon}(\Omega)}} & \lesssim_{\epsilon} h_{k}^{(\pi / \omega)-\epsilon}\left|R_{k}^{m}\left(z-z_{m}\right)\right|_{H^{1}(\Omega)} \\
& \lesssim_{\epsilon} m^{[-(\pi / \omega)+\epsilon] / 2}\left\|z-z_{0}\right\|_{H^{\alpha_{\epsilon}}(\Omega)} .
\end{aligned}
$$

Now we estimate the error for the $k^{\text {th }}$ level iteration. Let $\gamma_{m}=m^{[-(\pi / \omega)+\epsilon] / 2}$, and suppose that the error of the $(k-1)^{\text {st }}$ level iteration in the $H^{\alpha_{\epsilon}}(\Omega)$ norm is reduced by a factor $\eta$. Then it follows from (2.9) and (3.23) that

$$
\begin{aligned}
\left\|z-M G\left(k, z_{0}, g\right)\right\|_{H^{\alpha_{\epsilon}(\Omega)}} & \leq\|z-\tilde{z}\|_{H^{\alpha_{\epsilon}(\Omega)}}+\left\|q-q_{2}\right\|_{H^{\alpha_{\epsilon}(\Omega)}} \\
& \leq C_{\epsilon} \gamma_{m}\left\|z-z_{0}\right\|_{H^{\alpha_{\epsilon}(\Omega)}}+\eta^{2}\|q\|_{H^{\alpha_{\epsilon}(\Omega)}} .
\end{aligned}
$$

From (3.8), (3.11) and (3.17), we have

$$
\begin{aligned}
\left\|P_{k-1} v\right\|_{H^{\alpha_{\epsilon}(\Omega)}} & \leq\left\|v-P_{k-1} v\right\|_{H^{\alpha_{\epsilon}(\Omega)}}+\|v\|_{H^{\alpha_{\epsilon}(\Omega)}} \\
& \lesssim_{\epsilon} h_{k}^{(\pi / \omega)-\epsilon}|v|_{H^{1}(\Omega)}+\|v\|_{\alpha_{\epsilon}} \\
& \lesssim_{\epsilon}\|v\|_{\alpha_{\epsilon}} \quad \forall v \in V_{k} .
\end{aligned}
$$

Combining (3.5), (3.9) and (3.25), we obtain

$$
\begin{aligned}
\|q\|_{H^{\alpha_{\epsilon}(\Omega)}} & =\left\|P_{k-1}\left(z-z_{m}\right)\right\|_{H^{\alpha_{\epsilon}(\Omega)}} \\
& \leq C_{\epsilon}^{\prime}\left\|z-z_{0}\right\|_{\alpha_{\epsilon}} .
\end{aligned}
$$

The estimates (3.22), (3.24) and (3.26) together imply that

$$
\left\|z-M G\left(k, z_{0}, g\right)\right\|_{H^{\alpha_{\epsilon}(\Omega)}} \leq\left(C_{\epsilon} \gamma_{m}+C_{\epsilon}^{\prime \prime} \eta^{2}\right)\left\|z-z_{0}\right\|_{H^{\alpha_{\epsilon}(\Omega)}} .
$$

For $m$ sufficiently large, we have $\gamma_{m}<\left(4 C_{\epsilon} C_{\epsilon}^{\prime \prime}\right)^{-1}$ and

$$
\eta_{m}=\left[1-\left(1-4 C_{\epsilon} C_{\epsilon}^{\prime \prime} \gamma_{m}\right)^{1 / 2}\right] /\left(2 C_{\epsilon}^{\prime \prime}\right)
$$


is a fixed point of the map $T(\eta)=C_{\epsilon} \gamma_{m}+C_{\epsilon}^{\prime \prime} \eta^{2}$. Since the first level iteration is an exact solver, it follows from (3.27) and mathematical induction that

$$
\left\|z-M G\left(k, z_{0}, g\right)\right\|_{H^{\alpha_{\epsilon}(\Omega)}} \leq \eta_{m}\left\|z-z_{0}\right\|_{H^{\alpha_{\epsilon}}(\Omega)} \quad \text { for } \quad k \geq 1 .
$$

As $\lim _{m \rightarrow \infty} \eta_{m}=0$, the lemma follows from (3.28).

\section{Convergence ANALysis For the FUll MUltigrid Algorithm I}

Theorem 4.1. Let $p=2,0<\epsilon<\pi / \omega, \alpha_{\epsilon}=1-(\pi / \omega)+\epsilon \neq 1 / 2$ and the number of smoothing steps $m$ be sufficiently large so that (3.1) and (3.2) hold for $0<\delta<1$. If the number of nested iterations $n$ is sufficiently large, then we have

$$
\begin{aligned}
&\left|w-w_{k}\right|_{H^{1}(\Omega)} \lesssim h_{k}\|f\|_{L^{2}(\Omega)}, \\
& \sum_{j=1}^{J}\left|\kappa_{j}-\kappa_{j, k}\right| \lesssim \epsilon h_{k}^{1+(\pi / \omega)-\epsilon}\|f\|_{L^{2}(\Omega)}, \\
&\left\|w-w_{k}\right\|_{H^{\alpha \epsilon}(\Omega)} \lesssim \epsilon \\
& h_{k}^{1+(\pi / \omega)-\epsilon}\|f\|_{L^{2}(\Omega)},
\end{aligned}
$$

where $w_{k}$ and $\kappa_{j, k}$ are computed by (2.11)-(2.18).

Proof. We will establish (4.2) and (4.3) through recursive estimates. It follows from (1.5) and (2.15) that, for $1 \leq j \leq J$

$$
\begin{aligned}
\left|\kappa_{j}-\kappa_{j, k}\right| & =\frac{1}{\pi}\left|\int_{\Omega}\left(u-u_{k-1}\right) \Delta s_{-j} d x\right| \\
& \lesssim\left\|u-u_{k-1}\right\|_{L^{2}(\Omega)}\left\|\Delta s_{-j}\right\|_{L^{2}(\Omega)} \\
& \lesssim\left\|\hat{w}_{k-1}-w_{k-1}\right\|_{H^{\alpha_{\epsilon}(\Omega)}},
\end{aligned}
$$

where (cf. (1.2), (1.15) and (2.18))

$$
\hat{w}_{k}=u-\sum_{j=1}^{J} \kappa_{j, k} s_{j}=\sum_{j=1}^{J}\left(\kappa_{j}-\kappa_{j, k}\right) s_{j}+w=\left(u-u_{k}\right)+w_{k} .
$$

Let $a_{k}=\sum_{j=1}^{J}\left|\kappa_{j}-\kappa_{j, k}\right|$ and $b_{k}=\left\|\hat{w}_{k}-w_{k}\right\|_{H^{\alpha_{\epsilon}(\Omega)}}$. We have proved that

$$
a_{k} \lesssim b_{k-1} \text {. }
$$

To estimate $b_{k}$, we begin with

$$
b_{k} \leq\left\|\hat{w}_{k}-P_{k} \hat{w}_{k}\right\|_{H^{\alpha_{\epsilon}(\Omega)}}+\left\|P_{k} \hat{w}_{k}-w_{k}\right\|_{H^{\alpha_{\epsilon}(\Omega)}} .
$$

Using (1.4), (3.11), (4.5) and standard finite element discretization error estimates (cf. [20], [16]), we find

$$
\begin{aligned}
\| \hat{w}_{k} & -P_{k} \hat{w}_{k} \|_{H^{\alpha_{\epsilon}(\Omega)}} \\
& \leq \sum_{j=1}^{J}\left|\kappa_{j}-\kappa_{j, k}\right|\left\|s_{j}-P_{k} s_{j}\right\|_{H^{\alpha_{\epsilon}(\Omega)}}+\left\|w-P_{k} w\right\|_{H^{\alpha_{\epsilon}(\Omega)}} \\
& \lesssim_{\epsilon} h_{k}^{(\pi / \omega)-\epsilon}\left[\sum_{j=1}^{J}\left|\kappa_{j}-\kappa_{j, k}\right|\left|s_{j}-P_{k} s_{j}\right|_{H^{1}(\Omega)}+\left|w-P_{k} w\right|_{H^{1}(\Omega)}\right] \\
& \lesssim_{\epsilon} h_{k}^{2(\pi / \omega)-2 \epsilon} a_{k}+h_{k}^{1+(\pi / \omega)-\epsilon}\|f\|_{L^{2}(\Omega)} .
\end{aligned}
$$


Since $P_{k} \hat{w}_{k} \in V_{k}$ is the exact solution of the equation $A_{k} z=g_{k}$ (where $g_{k}$ is defined by (2.17)), it follows from (2.16) and (3.2) that

$$
\left\|P_{k} \hat{w}_{k}-w_{k}\right\|_{H^{\alpha_{\epsilon}(\Omega)}} \leq \delta^{n}\left\|P_{k} \hat{w}_{k}-w_{k-1}\right\|_{H^{\alpha_{\epsilon}(\Omega)}} .
$$

Note that (1.2) and (4.5) imply

$$
w-\hat{w}_{k}=\sum_{j=1}^{J}\left(\kappa_{j, k}-\kappa_{j}\right) s_{j} .
$$

From (4.8) and (4.10), we obtain

$$
\begin{aligned}
\left\|P_{k} \hat{w}_{k}-w_{k-1}\right\|_{H^{\alpha_{\epsilon}(\Omega)}} \leq & \left\|P_{k} \hat{w}_{k}-\hat{w}_{k}\right\|_{H^{\alpha_{\epsilon}(\Omega)}}+\left\|\hat{w}_{k}-w\right\|_{H^{\alpha_{\epsilon}(\Omega)}} \\
& +\left\|w-\hat{w}_{k-1}\right\|_{H^{\alpha_{\epsilon}}(\Omega)}+\left\|\hat{w}_{k-1}-w_{k-1}\right\|_{H^{\alpha_{\epsilon}(\Omega)}} \\
& \lesssim_{\epsilon} h_{k}^{2(\pi / \omega)-2 \epsilon} a_{k}+h_{k}^{1+(\pi / \omega)-\epsilon}\|f\|_{L^{2}(\Omega)}+a_{k}+a_{k-1}+b_{k-1} .
\end{aligned}
$$

It follows from (4.7)-(4.9) and (4.11) that

$$
b_{k} \lesssim \epsilon \delta^{n}\left(b_{k-1}+a_{k}+a_{k-1}\right)+h_{k}^{2(\pi / \omega)-2 \epsilon} a_{k}+h_{k}^{1+(\pi / \omega)-\epsilon}\|f\|_{L^{2}(\Omega)} .
$$

Combining (4.6) and (4.12), we find

$$
\left[\begin{array}{l}
a_{k} \\
b_{k}
\end{array}\right] \leq C_{\epsilon}\left[\begin{array}{cc}
0 & 1 \\
\delta^{n} & \delta^{n}+h_{k}^{2(\pi / \omega)-2 \epsilon}
\end{array}\right]\left[\begin{array}{l}
a_{k-1} \\
b_{k-1}
\end{array}\right]+C_{\epsilon} h_{k}^{1+(\pi / \omega)-\epsilon}\|f\|_{L^{2}(\Omega)}\left[\begin{array}{l}
0 \\
1
\end{array}\right]
$$

where the vector inequality is interpreted componentwise.

Since $\delta^{n}$ and $h_{k}^{2(\pi / \omega)-2 \epsilon}$ are both small for $n$ and $k$ large, we can consider the matrix in (4.13) to be a perturbation of the nilpotent matrix $\left[\begin{array}{cc}0 & C_{\epsilon} \\ 0 & 0\end{array}\right]$. Therefore, for $n$ sufficiently large and $k>k_{\epsilon}$, we have

$$
\left[\begin{array}{l}
a_{k} \\
b_{k}
\end{array}\right] \leq \mathbf{C}\left[\begin{array}{l}
a_{k-1} \\
b_{k-1}
\end{array}\right]+C_{\epsilon} h_{k}^{1+(\pi / \omega)-\epsilon}\|f\|_{L^{2}(\Omega)}\left[\begin{array}{l}
0 \\
1
\end{array}\right]
$$

where the constant matrix $\mathbf{C}$ has nonnegative components and satisfies

$$
\left\|\mathbf{C}^{n}\right\|_{\infty} \lesssim \frac{1}{4^{n}}
$$

By iterating (4.14), for $k>k_{\epsilon}$, we obtain

$$
\begin{aligned}
\left\|\left[\begin{array}{l}
a_{k} \\
b_{k}
\end{array}\right]\right\|_{\infty} \lesssim & \left(h_{k}^{1+(\pi / \omega)-\epsilon}+\frac{1}{4} h_{k-1}^{1+(\pi / \omega)-\epsilon}+\frac{1}{4^{2}} h_{k-2}^{1+(\pi / \omega)-\epsilon}+\cdots\right)\|f\|_{L^{2}(\Omega)} \\
& +\left(\frac{1}{4}\right)^{k-k_{\epsilon}}\left\|\left[\begin{array}{l}
a_{k_{\epsilon}} \\
b_{k_{\epsilon}}
\end{array}\right]\right\|_{\infty} .
\end{aligned}
$$

Since $h_{k-1}=2 h_{k}$ and $1+(\pi / \omega)-\epsilon<2$, it follows from (4.16) that

$$
\left\|\left[\begin{array}{l}
a_{k} \\
b_{k}
\end{array}\right]\right\|_{\infty} \lesssim_{\epsilon} h_{k}^{1+(\pi / \omega)-\epsilon}\|f\|_{L^{2}(\Omega)}+\left(\frac{1}{4}\right)^{k-k_{\epsilon}}\left\|\left[\begin{array}{l}
a_{k_{\epsilon}} \\
b_{k_{\epsilon}}
\end{array}\right]\right\|_{\infty} .
$$

On the other hand, from (1.4) and (2.11)-(2.14) we have

$$
\begin{aligned}
a_{1} & =\sum_{j=1}^{J}\left|\kappa_{j}\right| \lesssim\|f\|_{L^{2}(\Omega)}, \\
b_{1} & =\left\|u-P_{1} u\right\|_{H^{\alpha_{\epsilon}(\Omega)}} \lesssim\|u\|_{H^{1}(\Omega)} \lesssim\|f\|_{L^{2}(\Omega)} .
\end{aligned}
$$


It follows from (4.13), (4.18) and (4.19) that

$$
\left\|\left[\begin{array}{l}
a_{k} \\
b_{k}
\end{array}\right]\right\|_{\infty} \lesssim_{\epsilon}\|f\|_{L^{2}(\Omega)} \lesssim_{\epsilon} h_{k}^{1+(\pi / \omega)-\epsilon}\|f\|_{L^{2}(\Omega)} \quad \text { for } \quad 1 \leq k \leq k_{\epsilon} .
$$

Combining (4.17) and (4.20), we find

$$
\left\|\left[\begin{array}{l}
a_{k} \\
b_{k}
\end{array}\right]\right\|_{\infty} \lesssim \epsilon h_{k}^{1+(\pi / \omega)-\epsilon}\|f\|_{L^{2}(\Omega)} \quad \text { for } \quad k \geq 1 .
$$

We have established (4.2), and the estimate (4.3) follows from (4.10) and (4.21).

Repeating the arguments in (4.7)-(4.11) with respect to the $|\cdot|_{H^{1}(\Omega)}$ norm and using (4.2) with an $\epsilon<\pi / \omega$, we find

$$
\left|\hat{w}_{k}-w_{k}\right|_{H^{1}(\Omega)} \lesssim \delta^{n}\left|\hat{w}_{k-1}-w_{k-1}\right|_{H^{1}(\Omega)}+h_{k}\|f\|_{L^{2}(\Omega)} .
$$

We obtain from iterating (4.22) that

$$
\left|\hat{w}_{k}-w_{k}\right|_{H^{1}(\Omega)} \lesssim h_{k}\|f\|_{L^{2}(\Omega)}
$$

The estimate (4.1) follows from (4.2) (with $\epsilon<\pi / \omega)$, (4.10) and (4.23).

The following corollary is immediate.

Corollary 4.2. Under the assumptions of Theorem 4.1, we have

$$
\left|u-u_{k}\right|_{H^{1}(\Omega)} \lesssim h_{k}\|f\|_{L^{2}(\Omega)} .
$$

\section{CONVERGEnce ANAlysis FOR the FUll MUltigRid ALGORIthm II}

Since we are really computing the regular part of the singular solution in our approach, it is possible to exploit superconvergence results. This idea will be demonstrated by the following analysis of the full multigrid algorithm II.

We first investigate the properties of the intergrid transfer operator $I_{k-1}^{k}$.

Lemma 5.1. The following estimates hold for the intergrid transfer operator defined by (2.27):

$$
\begin{aligned}
\left|I_{k-1}^{k} v\right|_{H^{1}(\Omega)} & \lesssim|v|_{H^{1}(\Omega)} \quad \forall v \in V_{k-1}, \\
\left|\Pi_{k} \zeta-I_{k-1}^{k} \Pi_{k-1} \zeta\right|_{H^{1}(\Omega)} & \lesssim h_{k}^{1+t}\|\zeta\|_{H^{2+t}(\Omega)} \quad \forall \zeta \in H^{2+t}(\Omega) \cap H_{0}^{1}(\Omega),
\end{aligned}
$$

where $\Pi_{k}$ is the nodal interpolation operator associated with $V_{k}$ and $0 \leq t \leq 1$.

Proof. Let $T$ be a triangle, $\eta$ be a quadratic polynomial, and $\eta^{I}$ be the linear interpolant of $\eta$ with respect to the subdivision of $T$ obtained by connecting the midpoints of the sides of $T$. A standard scaling argument shows that

$$
C_{1}\left|\eta^{I}\right|_{H^{1}(T)} \leq|\eta|_{H^{1}(T)} \leq C_{2}\left|\eta^{I}\right|_{H^{1}(T)},
$$

where the constants $C_{1}$ and $C_{2}$ depend only on the angles of $T$.

Recall that $Q_{k} \subset H_{0}^{1}(\Omega)(k=0,1,2, \ldots)$ is the quadratic Lagrange finite element space associated with $\mathcal{T}_{k}$, and $\mathcal{I}_{k-2}^{k-1}$ (resp., $\mathcal{I}_{k-2}^{k}$ ) is the nodal interpolation operator from $Q_{k-2}$ into $V_{k-1}$ (resp., $V_{k}$ ). We deduce from (5.3) that, for $k \geq 2$,

$$
\left|\mathcal{I}_{k-2}^{k} q\right|_{H^{1}(\Omega)} \lesssim|q|_{H^{1}(\Omega)} \lesssim\left|\mathcal{I}_{k-2}^{k-1} q\right|_{H^{1}(\Omega)} \quad \forall q \in Q_{k-2} .
$$

The estimate (5.1) follows from (2.27) and (5.4). 


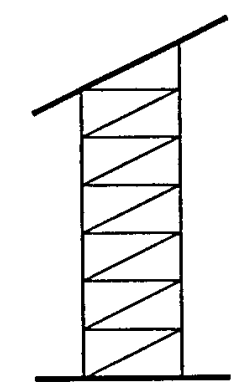

FiguRE 1

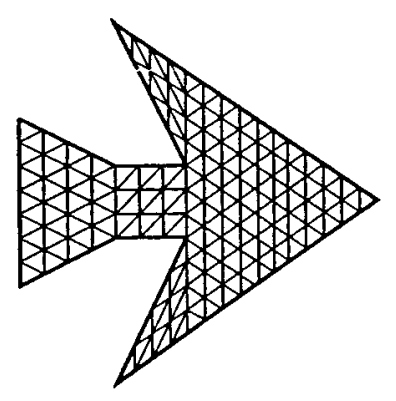

FiguRE 2

Let $T$ be a triangle in $\mathcal{T}_{k-2}$ and $q$ be a polynomial of degree $\leq 2$. By restricting all the definitions to $T$, we find

$$
\begin{aligned}
\Pi_{k} q-I_{k-1}^{k} \Pi_{k-1} q & =\Pi_{k} q-\mathcal{I}_{k-2}^{k}\left(\mathcal{I}_{k-2}^{k-1}\right)^{-1} \Pi_{k-1} q \\
& =\Pi_{k} q-\mathcal{I}_{k-2}^{k} q=0 .
\end{aligned}
$$

By the Bramble-Hilbert lemma (cf. [11]) and a standard scaling argument we deduce from (5.5) that

$$
\begin{aligned}
& \left|\Pi_{k} \zeta-I_{k-1}^{k} \Pi_{k-1} \zeta\right|_{H^{1}(\Omega)} \lesssim h_{k}^{2}\|\zeta\|_{H^{3}(\Omega)} \quad \forall \zeta \in H^{3}(\Omega) \cap H_{0}^{1}(\Omega), \\
& \left|\Pi_{k} \zeta-I_{k-1}^{k} \Pi_{k-1} \zeta\right|_{H^{1}(\Omega)} \lesssim h_{k}\|\zeta\|_{H^{2}(\Omega)} \quad \forall \zeta \in H^{2}(\Omega) \cap H_{0}^{1}(\Omega) .
\end{aligned}
$$

The estimate (5.2) follows from interpolating (5.6) and (5.7) (cf. [50]).

Next we discuss a superconvergence result for the linear finite element. A uniform band in a triangulation is a collection of triangles between two parallel lines such that any two triangles sharing a common side form a parallelogram (cf. Figure 1), and the boundary of the band consists of the two parallel lines and parts of $\partial \Omega$. We say that a triangulation satisfies the uniform band condition if it can be divided completely into uniform bands (cf. Figure 2). Note that one can always find a triangulation satisfying the uniform band condition for any polygonal domain whose vertices all have rational coordinates, and the uniform band condition is preserved by regular subdivision.

The proof of the following result can be found in [18] (cf. also [34], [41], [61], [19], [35]). 
Lemma 5.2. Suppose the triangulations $\mathcal{T}_{k}$ satisfy the uniform band condition and $\zeta \in H^{3}(\Omega) \cap H_{0}^{1}(\Omega)$. Then

$$
\left|\Pi_{k} \zeta-P_{k} \zeta\right|_{H^{1}(\Omega)} \lesssim h_{k}^{2}\|\zeta\|_{H^{3}(\Omega)} .
$$

Corollary 5.3. Suppose the triangulations $\mathcal{T}_{k}$ satisfy the uniform band condition and $\zeta \in H^{2+t}(\Omega) \cap H_{0}^{1}(\Omega)$ for $0 \leq t \leq 1$. Then

$$
\left|\Pi_{k} \zeta-P_{k} \zeta\right|_{H^{1}(\Omega)} \lesssim h_{k}^{1+t}\|\zeta\|_{H^{2+t}(\Omega)} .
$$

Proof. The estimate (5.9) follows from interpolating (5.8) and the obvious estimate

$$
\left|\Pi_{k} \zeta-P_{k} \zeta\right|_{H^{1}(\Omega)} \lesssim h_{k}\|\zeta\|_{H^{2}(\Omega)} \quad \forall \zeta \in H^{2}(\Omega) \cap H_{0}^{1}(\Omega) .
$$

We now establish the convergence properties of the full multigrid algorithm II.

Theorem 5.4. Let $f \in H^{1}(\Omega)$. Assume that the triangulations $\mathcal{T}_{k}$ satisfy the uniform band condition, $p=1$ or 2 , and $m \geq 1$. If the number of nested iterations $n$ is sufficiently large, then we have

$$
\begin{aligned}
\left|\Pi_{k} w-w_{k}\right|_{H^{1}(\Omega)} & \lesssim \epsilon h_{k}^{2-\epsilon}\|f\|_{H^{1}(\Omega)}, \\
\sum_{j=1}^{N} \sum_{\ell \in \mathcal{L}_{j}}\left|\kappa_{j, \ell}-\kappa_{j, \ell, k}\right| & \lesssim \epsilon h_{k}^{2-\epsilon}\|f\|_{H^{1}(\Omega)},
\end{aligned}
$$

where $w_{k}$ and $\kappa_{j, \ell, k}$ are computed by $(2.19)-(2.26)$.

Proof. It follows from (1.24), (2.23), (2.26) and the Poincaré inequality that

$$
\begin{aligned}
\left|\kappa_{j, \ell}-\kappa_{j, \ell, k}\right| & =\frac{1}{\ell \pi}\left|\int_{\Omega}\left(\hat{w}_{k-1}-w_{k-1}\right) \Delta s_{j,-\ell} d x\right| \\
\lesssim & \left|\int_{\Omega}\left(\hat{w}_{k-1}-\Pi_{k-1} \hat{w}_{k-1}\right) \Delta s_{j,-\ell} d x\right| \\
& \quad+\left|\int_{\Omega}\left(\Pi_{k-1} \hat{w}_{k-1}-w_{k-1}\right) \Delta s_{j,-\ell} d x\right| \\
\lesssim & \left\|\hat{w}_{k-1}-\Pi_{k-1} \hat{w}_{k-1}\right\|_{L^{2}(\Omega)}+\left|\Pi_{k-1} \hat{w}_{k-1}-w_{k-1}\right|_{H^{1}(\Omega)},
\end{aligned}
$$

where

$$
\begin{aligned}
\hat{w}_{k} & =u-\sum_{j=1}^{N} \sum_{\ell \in \mathcal{L}_{j}} \kappa_{j, \ell, k} s_{j, \ell}=\left(u-u_{k}\right)+w_{k} \\
& =\sum_{j=1}^{N} \sum_{\ell \in \mathcal{L}_{j}}\left(\kappa_{j, \ell}-\kappa_{j, \ell, k}\right) s_{j, \ell}+w .
\end{aligned}
$$

Let $a_{k}=\sum_{j=1}^{N} \sum_{\ell \in \mathcal{L}_{j}}\left|\kappa_{j, \ell}-\kappa_{j, \ell, k}\right|$ and $b_{k}=\left|\Pi_{k} \hat{w}_{k}-w_{k}\right|_{H^{1}(\Omega)}$. Since $s_{j, \ell} \in$ $H^{1+(\pi / \omega)-\epsilon}(\Omega)$ and $w \in H^{3-\epsilon}(\Omega)$, we have from (1.23), (5.13) and standard interpolation error estimates that

$$
\begin{aligned}
\left\|\hat{w}_{k-1}-\Pi_{k-1} \hat{w}_{k-1}\right\|_{L^{2}(\Omega)} \leq & \sum_{j=1}^{N} \sum_{\ell \in \mathcal{L}_{j}}\left|\kappa_{j, \ell}-\kappa_{j, \ell, k-1}\right|\left\|s_{j, \ell}-\Pi_{k-1} s_{j, \ell}\right\|_{L^{2}(\Omega)} \\
& +\left\|w-\Pi_{k-1} w\right\|_{L^{2}(\Omega)} \\
& \lesssim_{\epsilon} h_{k-1}^{1+(\pi / \omega)-\epsilon} a_{k-1}+h_{k-1}^{2}\|f\|_{H^{1}(\Omega)} .
\end{aligned}
$$


It follows from (5.12) and (5.14) that

$$
a_{k} \lesssim{ }_{\epsilon} h_{k}^{1+(\pi / \omega)-\epsilon} a_{k-1}+b_{k-1}+h_{k}^{2}\|f\|_{H^{1}(\Omega)} .
$$

On the other hand, we have

$$
b_{k} \leq\left|\Pi_{k} \hat{w}_{k}-P_{k} \hat{w}_{k}\right|_{H^{1}(\Omega)}+\left|P_{k} \hat{w}_{k}-w_{k}\right|_{H^{1}(\Omega)},
$$

and from (1.23), (5.9), (5.13) and a standard interpolation error estimate,

$$
\begin{aligned}
&\left|\Pi_{k} \hat{w}_{k}-P_{k} \hat{w}_{k}\right|_{H^{1}(\Omega)} \leq \sum_{j=1}^{N} \sum_{\ell \in \mathcal{L}_{j}}\left|\kappa_{j, \ell}-\kappa_{j, \ell, k}\right|\left|\Pi_{k} s_{j, \ell}-P_{k} s_{j, \ell}\right|_{H^{1}(\Omega)} \\
& \quad+\left|\Pi_{k} w-P_{k} w\right|_{H^{1}(\Omega)} \\
& \lesssim_{\epsilon} h_{k}^{(\pi / \omega)-\epsilon} a_{k}+h_{k}^{2-\epsilon}\|f\|_{H^{1}(\Omega)} .
\end{aligned}
$$

Since $P_{k} \hat{w}_{k} \in V_{k}$ is the exact solution of $A_{k} z=g_{k}$ (where $g_{k}$ is defined by (2.25)), it follows from (2.24) and Lemma 3.1 that

$$
\left|P_{k} \hat{w}_{k}-w_{k}\right|_{H^{1}(\Omega)} \leq \delta^{n}\left|P_{k} \hat{w}_{k}-I_{k-1}^{k} w_{k-1}\right|_{H^{1}(\Omega)},
$$

for some $\delta<1$.

It follows from (1.23), (5.1), (5.2) and (5.13) that

$$
\begin{aligned}
& \left|P_{k} \hat{w}_{k}-I_{k-1}^{k} w_{k-1}\right|_{H^{1}(\Omega)} \\
& \quad \leq\left|P_{k}\left(\hat{w}_{k}-w\right)\right|_{H^{1}(\Omega)}+\left|P_{k} w-\Pi_{k} w\right|_{H^{1}(\Omega)} \\
& \quad \quad \quad|| \Pi_{k} w-\left.I_{k-1}^{k} \Pi_{k-1} w\right|_{H^{1}(\Omega)}+\left|I_{k-1}^{k}\left(\Pi_{k-1} w-w_{k-1}\right)\right|_{H^{1}(\Omega)} \\
& \quad \lesssim_{\epsilon} a_{k}+h_{k}^{2-\epsilon}\|f\|_{H^{1}(\Omega)}+\left|\Pi_{k-1} w-w_{k-1}\right|_{H^{1}(\Omega)} \\
& \quad{ }_{\epsilon} a_{k}+h_{k}^{2-\epsilon}\|f\|_{H^{1}(\Omega)}+\left|\Pi_{k-1}\left(w-\hat{w}_{k-1}\right)\right|_{H^{1}(\Omega)} \\
& \quad+\left|\Pi_{k-1} \hat{w}_{k-1}-w_{k-1}\right|_{H^{1}(\Omega)} \\
& \quad \lesssim_{\epsilon} a_{k}+a_{k-1}+b_{k-1}+h_{k}^{2-\epsilon}\|f\|_{H^{1}(\Omega)} .
\end{aligned}
$$

Using (5.16)-(5.19) we obtain

$$
b_{k} \lesssim \epsilon\left(h_{k}^{(\pi / \omega)-\epsilon}+\delta^{n}\right) a_{k}+\delta^{n}\left(a_{k-1}+b_{k-1}\right)+h_{k}^{2-\epsilon}\|f\|_{H^{1}(\Omega)} .
$$

As in the proof of Theorem 4.1, by iterating (5.15) and (5.20) we have

$$
\begin{aligned}
& a_{k} \lesssim_{\epsilon} h_{k}^{2-\epsilon}\|f\|_{H^{1}(\Omega)}, \\
& b_{k} \lesssim \epsilon h_{k}^{2-\epsilon}\|f\|_{H^{1}(\Omega)} .
\end{aligned}
$$

The estimate (5.11) is the same as (5.21). We deduce from (5.13) that

$$
\begin{aligned}
\left|\Pi_{k} w-w_{k}\right|_{H^{1}(\Omega)} & \leq\left|\Pi_{k}\left(w-\hat{w}_{k}\right)\right|_{H^{1}(\Omega)}+\left|\Pi_{k} \hat{w}_{k}-w_{k}\right|_{H^{1}(\Omega)} \\
& \lesssim a_{k}+b_{k} .
\end{aligned}
$$

The estimate (5.10) follows from (5.21)-(5.23).

Remark. By exploiting superconvergence we are able to perform the analysis in the proof of Theorem 5.4 using the energy norm and still obtain a higher rate of convergence. The use of the energy norm also makes it possible to apply Lemma 3.1 (instead of Lemma 3.2) to demonstrate convergence even for the $V$-cycle algorithm with one smoothing step.

The following corollary is immediate. 
Corollary 5.5. Under the assumptions of Theorem 5.4, we have

$$
\left|u-u_{k}\right|_{H^{1}(\Omega)} \lesssim h_{k}\|f\|_{H^{1}(\Omega)} .
$$

Corollary 5.6. Under the assumptions of Theorem 5.4, we have

$$
\max _{p}\left|u(p)-u_{k}(p)\right| \lesssim_{\epsilon} h_{k}^{2-\epsilon}\|f\|_{H^{1}(\Omega)},
$$

where the maximum is taken over all the vertices $p$ of $\mathcal{T}_{k}$.

Proof. It suffices to estimate $\left\|\Pi_{k}\left(u-u_{k}\right)\right\|_{L^{\infty}(\Omega)}$. By (1.17), (2.26), (5.10), (5.11) and the discrete Sobolev inequality (cf. [46], [61]), we have

$$
\begin{aligned}
\left\|\Pi_{k}\left(u-u_{k}\right)\right\|_{L^{\infty}(\Omega)} & \leq \sum_{j=1}^{N} \sum_{\ell \in \mathcal{L}_{j}}\left|\kappa_{j, \ell}-\kappa_{j, \ell, k}\right|\left\|\Pi_{k} s_{j, \ell}\right\|_{L^{\infty}(\Omega)}+\left\|\Pi_{k} w-w_{k}\right\|_{L^{\infty}(\Omega)} \\
& \lesssim \sum_{j=1}^{N} \sum_{\ell \in \mathcal{L}_{j}}\left|\kappa_{j, \ell}-\kappa_{j, \ell, k}\right|+\left|\ln h_{k}\right|^{1 / 2}\left|\Pi_{k} w-w_{k}\right|_{H^{1}(\Omega)} \\
& \lesssim{ }_{\epsilon} h_{k}^{2-\epsilon}\|f\|_{H^{1}(\Omega)} .
\end{aligned}
$$

Remark. If none of the angles of $\Omega$ is an integer multiple of $\pi / 2$, then the regular part $w$ in (1.20) actually belongs to $H^{3}(\Omega)$ (cf. [27], [23]). For such $\Omega$ the $\epsilon$ dependence in (1.23), (5.9) and (5.10) disappears, and the estimate (5.25) becomes

$$
\max _{p}\left|u(p)-u_{k}(p)\right| \lesssim h_{k}^{2}\left|\ln h_{k}\right|^{1 / 2}\|f\|_{H^{1}(\Omega)} .
$$

Remark. It is well known (cf. [47], [44], [16]) that

$$
\left\|u-\tilde{u}_{h}\right\|_{L^{\infty}(\Omega)} \lesssim h^{2}|\ln h|\|u\|_{W_{\infty}^{2}(\Omega)},
$$

where $\tilde{u}_{h}$ is the P1 finite element solution of (1.1). It is interesting to see that a multigrid method can produce similar results for singular solutions.

\section{Numerical EXPERIments}

In this section, we report the results of some numerical experiments for (1.1) performed on the $\Gamma$-shaped domain $\Omega$ (cf. Figures 3 and 4 ) with vertices $(0,0)$, $(0,1),(1,1),(-1,1),(-1,-1)$ and $(0,-1)$. We use the P1 Lagrange finite element in the experiments. All the computations are done using a $W$-cycle $k^{\text {th }}$ level iteration with 5 smoothing steps, and the number of nested iterations in the full multigrid algorithms is also 5 . The mesh size $h_{k}$ for the $k^{\text {th }}$ level grid is taken to be $2^{-k}$. We first describe the experiments; comments on the numerical results are given near the end of this section.

Remark. Convergence of the algorithms has been observed for $m=n=1$. We choose to report the results for $m=n=5$, since the numerical results do not appear to improve for any larger $m$ or $n$.

The two singular functions for this $\Gamma$-shaped domain are:

$$
\begin{aligned}
& s_{1}(r, \theta)=\phi(r) r^{2 / 3} \sin ((2 / 3) \theta), \\
& s_{2}(r, \theta)=\phi(r) r^{4 / 3} \sin ((4 / 3) \theta),
\end{aligned}
$$




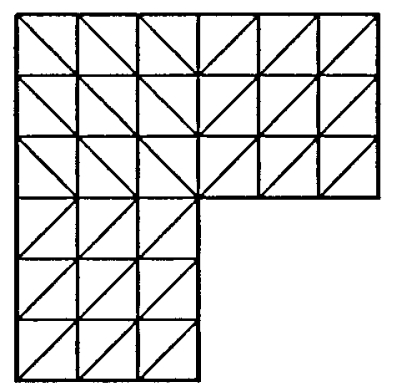

FIGURE 3

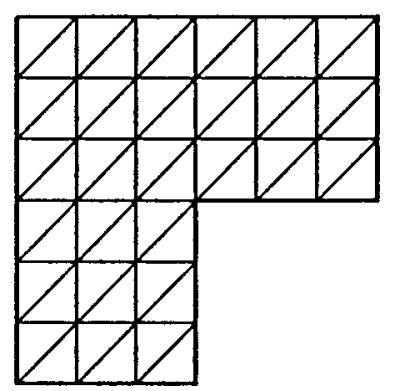

Figure 4

where the cut-off function $\phi(r)$ is defined by

$$
\phi(r)= \begin{cases}1 & 0 \leq r \leq 1 / 4, \\ -192 r^{5}+480 r^{4}-440 r^{3}+180 r^{2}-\frac{135}{4} r+\frac{27}{8} & 1 / 4 \leq r \leq 3 / 4, \\ 0 & 3 / 4 \leq r\end{cases}
$$

In the first set of experiments, we solve the Poisson equation (1.1) with $f=$ $-\Delta s_{1}-\Delta s_{2}+6 x\left(y^{2}-y^{4}\right)+\left(x-x^{3}\right)\left(12 y^{2}-2\right)$. The exact solution is therefore

$$
u=s_{1}+s_{2}+\left(x-x^{3}\right)\left(y^{2}-y^{4}\right) .
$$

In the first experiment, we solve (1.1) using the standard full multigrid algorithm (where the right-hand sides of the discrete equations on all levels come from $f$ ) on the grids depicted in Figure 3. The approximate stress intensity factors $\kappa_{k}$ are computed by the extraction formula (1.7) using the P1 finite element solution $u_{k}$ obtained by the standard full multigrid method. The error $e_{k}$ in the energy norm is defined by $e_{k}=\left|\Pi_{k} u-u_{k}\right|_{H^{1}(\Omega)}$. The rate of convergence $\sigma_{k}$ for the stress intensity factor and the rate of convergence $\epsilon_{k}$ in the energy norm are computed by

$$
\begin{aligned}
\sigma_{k} & =\log _{2}\left(\frac{\left|\kappa_{k-1}-1\right|}{\left|\kappa_{k}-1\right|}\right), \\
\epsilon_{k} & =\log _{2}\left(\frac{e_{k-1}}{e_{k}}\right) .
\end{aligned}
$$

The results are tabulated in Table 1.

In the second experiment, we solve (6.1) using the full multigrid algorithm I described in Section 2 on the grids depicted in Figure 3. This algorithm computes 
TABLE 1. Results for the standard full multigrid algorithm with $f=-\Delta s_{1}-\Delta s_{2}+6 x\left(y^{2}-y^{4}\right)+\left(x-x^{3}\right)\left(12 y^{2}-2\right)$

\begin{tabular}{|c|c|c|c|c|}
\hline$k$ & $\kappa_{k}$ & $\sigma_{k}$ & $e_{k}$ & $\epsilon_{k}$ \\
\hline 1 & 1.6999229601 & - & $1.27093 \times 10^{0}$ & - \\
\hline 2 & 1.2589102299 & 1.43 & $5.91072 \times 10^{-1}$ & 1.1045 \\
\hline 3 & 1.1036407706 & 1.32 & $1.61387 \times 10^{-1}$ & 1.8728 \\
\hline 4 & 1.0287080790 & 1.85 & $5.74371 \times 10^{-2}$ & 1.4905 \\
\hline 5 & 1.0073492045 & 1.97 & $2.76732 \times 10^{-2}$ & 1.0535 \\
\hline 6 & 1.0020544785 & 1.84 & $1.64752 \times 10^{-2}$ & 0.7482 \\
\hline 7 & 1.0005531037 & 1.89 & $1.02811 \times 10^{-2}$ & 0.6803 \\
\hline 8 & 1.0001571227 & 1.82 & $6.46930 \times 10^{-2}$ & 0.6683 \\
\hline 9 & 1.0000458701 & 1.78 & $4.07502 \times 10^{-3}$ & 0.6668 \\
\hline 10 & 1.0000142397 & 1.69 & $2.56715 \times 10^{-3}$ & 0.6666 \\
\hline 11 & 1.0000046460 & 1.62 & $1.61722 \times 10^{-3}$ & 0.6666 \\
\hline
\end{tabular}

TABLE 2. Results for the full multigrid algorithm I with $f=$ $-\Delta s_{1}-\Delta s_{2}+6 x\left(y^{2}-y^{4}\right)+\left(x-x^{3}\right)\left(12 y^{2}-2\right)$

\begin{tabular}{|c|c|c|c|c|}
\hline$k$ & $\kappa_{k}$ & $\sigma_{k}$ & $e_{k}$ & $\epsilon_{k}$ \\
\hline 1 & - & - & $7.929 \times 10^{-1}$ & - \\
\hline 2 & 1.69992296014 & - & $8.364 \times 10^{-1}$ & -0.07 \\
\hline 3 & 0.82132136706 & 1.97 & $2.322 \times 10^{-1}$ & 1.85 \\
\hline 4 & 1.02037630458 & 3.13 & $3.456 \times 10^{-2}$ & 2.75 \\
\hline 5 & 0.99943755129 & 5.18 & $6.236 \times 10^{-3}$ & 2.47 \\
\hline 6 & 1.00003984026 & 3.82 & $1.595 \times 10^{-3}$ & 1.97 \\
\hline 7 & 1.00000536058 & 2.89 & $4.200 \times 10^{-4}$ & 1.93 \\
\hline 8 & 1.00000234005 & 1.20 & $1.170 \times 10^{-4}$ & 1.84 \\
\hline 9 & 1.00000057569 & 2.02 & $3.567 \times 10^{-5}$ & 1.71 \\
\hline 10 & 1.00000012632 & 2.19 & $1.204 \times 10^{-5}$ & 1.57 \\
\hline 11 & 1.00000002876 & 2.13 & $4.397 \times 10^{-6}$ & 1.45 \\
\hline 12 & 1.00000000746 & 1.95 & - & - \\
\hline
\end{tabular}

$\kappa_{k} \in \mathbb{R}$ and $w_{k} \in V_{k}$ which are approximations of the stress intensity factor $\kappa=1$ and the regular part of the solution in (6.4), namely, $w=s_{2}+\left(x-x^{3}\right)\left(y^{2}-y^{4}\right)$. The error $e_{k}$ in the energy norm is defined by $e_{k}=\left|\Pi_{k} w-w_{k}\right|_{H^{1}(\Omega)}$. The convergence rates $\sigma_{k}$ and $\epsilon_{k}$ are again computed by (6.5) and (6.6). The results are tabulated in Table 2 .

In the third experiment, we solve (1.1) by the full multigrid algorithm II described in Section 2 on the uniform grids depicted in Figure 4. This algorithm computes $\kappa_{1, k} \in \mathbb{R}, \kappa_{2, k} \in \mathbb{R}$ and $w_{k} \in V_{k}$ which are approximations of the stress intensity factors $\kappa_{1}=1, \kappa_{2}=1$ and the regular part of the solution in (6.4), namely, $w=$ $\left(x-x^{3}\right)\left(y^{2}-y^{4}\right)$. The error $e_{k}$ in the energy norm is defined by $e_{k}=\left|\Pi_{k} w-w_{k}\right|_{H^{1}(\Omega)}$ The convergence rates $\sigma_{1, k}\left(\sigma_{2, k}\right)$ and $\epsilon_{k}$ are computed as in (6.5) and (6.6). The results are tabulated in Table 3 . 
TABLE 3. Results for the full multigrid algorithm II with $f=$ $-\Delta s_{1}-\Delta s_{2}+6 x\left(y^{2}-y^{4}\right)+\left(x-x^{3}\right)\left(12 y^{2}-2\right)$

\begin{tabular}{|c|c|r|c|c|c|c|}
\hline$k$ & $\kappa_{1, k}$ & $\sigma_{1, k}$ & $\kappa_{2, k}$ & $\sigma_{2, k}$ & $e_{k}$ & $\epsilon_{k}$ \\
\hline 1 & - & - & - & - & $1.124 \times 10^{0}$ & - \\
\hline 2 & 1.6229151283 & - & 1.17131298888 & - & $7.361 \times 10^{-1}$ & 0.61 \\
\hline 3 & 0.8859991798 & 2.45 & 0.99336080108 & 4.69 & $1.417 \times 10^{-1}$ & 2.38 \\
\hline 4 & 1.0091773397 & 3.63 & 1.00029662538 & 4.48 & $1.131 \times 10^{-2}$ & 3.65 \\
\hline 5 & 0.9999856171 & 9.32 & 1.00023130682 & 0.36 & $5.829 \times 10^{-4}$ & 4.28 \\
\hline 6 & 1.0000653041 & -2.18 & 1.00002651087 & 3.13 & $1.551 \times 10^{-4}$ & 1.91 \\
\hline 7 & 1.0000136298 & 2.26 & 1.00000976600 & 1.44 & $3.636 \times 10^{-5}$ & 2.09 \\
\hline 8 & 1.0000044994 & 1.60 & 1.00000116447 & 3.07 & $9.574 \times 10^{-6}$ & 1.93 \\
\hline 9 & 1.0000011279 & 2.00 & 1.00000029598 & 1.98 & $2.376 \times 10^{-6}$ & 2.01 \\
\hline 10 & 1.0000002659 & 2.08 & 1.00000008791 & 1.75 & $5.810 \times 10^{-7}$ & 2.03 \\
\hline 11 & 1.0000000638 & 2.06 & 1.00000002475 & 1.82 & $1.433 \times 10^{-7}$ & 2.02 \\
\hline 12 & 1.0000000163 & 1.97 & 1.00000000585 & 2.08 & - & - \\
\hline
\end{tabular}

TABLE 4. Results for the standard full multigrid algorithm with $f=1$

\begin{tabular}{|c|c|r|c|c|}
\hline$k$ & $\kappa_{k}$ & \multicolumn{1}{|c|}{$\sigma_{k}$} & $e_{k}$ & $\epsilon_{k}$ \\
\hline 1 & 0.40404781 & - & $2.513 \times 10^{-1}$ & - \\
\hline 2 & 0.40105510 & 2.46 & $1.378 \times 10^{-1}$ & 0.866 \\
\hline 3 & 0.40051009 & -2.07 & $7.465 \times 10^{-2}$ & 0.885 \\
\hline 4 & 0.40280457 & 1.62 & $4.104 \times 10^{-2}$ & 0.863 \\
\hline 5 & 0.40205736 & 6.72 & $2.313 \times 10^{-2}$ & 0.827 \\
\hline 6 & 0.40205027 & -3.50 & $1.338 \times 10^{-2}$ & 0.790 \\
\hline 7 & 0.40197036 & 1.87 & $7.919 \times 10^{-3}$ & 0.756 \\
\hline 8 & 0.40194849 & 1.08 & $4.776 \times 10^{-3}$ & 0.729 \\
\hline 9 & 0.40193815 & 1.29 & $2.922 \times 10^{-3}$ & 0.709 \\
\hline 10 & 0.40193393 & 1.28 & $1.805 \times 10^{-3}$ & 0.695 \\
\hline 11 & 0.40193219 & - & - & - \\
\hline
\end{tabular}

In the second set of experiments, we solve the Poisson equation

$$
\begin{aligned}
-\Delta u=1 & \text { in } \quad \Omega, \\
u=0 & \text { on } \quad \partial \Omega .
\end{aligned}
$$

In the fourth experiment, we apply the standard full multigrid algorithm to (6.7) on the grids in Figure 3. The error $e_{k}$ in the energy norm is defined by $e_{k}=\left|u_{k}-u_{k+1}\right|_{H^{1}(\Omega)}$. The convergence rates $\epsilon_{k}$ and $\sigma_{k}$ are computed by (6.6) and

$$
\sigma_{k}=\log _{2}\left(\frac{\left|\kappa_{k}-\kappa_{k-1}\right|}{\left|\kappa_{k}-\kappa_{k+1}\right|}\right) .
$$

The results are tabulated in Table 4 .

In the fifth experiment, we apply the full multigrid algorithm I to (6.7) on the grids depicted in Figure 3. The error $e_{k}$ in energy norm is now defined by $e_{k}=$ $\left|w_{k}-w_{k+1}\right|_{H^{1}(\Omega)}$. The convergence rates $\sigma_{k}$ and $\epsilon_{k}$ are computed by (6.8) and (6.6) respectively. The results are tabulated in Table 5 . 
TABLE 5. Results for the full multigrid algorithm I with $f=1$

\begin{tabular}{|c|c|c|c|c|}
\hline$k$ & $\kappa_{k}$ & $\sigma_{k}$ & $e_{k}$ & $\epsilon_{k}$ \\
\hline 1 & - & - & $4.138 \times 10^{-1}$ & - \\
\hline 2 & 0.40404781 & - & $2.565 \times 10^{-1}$ & 0.690 \\
\hline 3 & 0.29704637 & 0.55 & $1.603 \times 10^{-1}$ & 0.678 \\
\hline 4 & 0.37020221 & 1.73 & $8.073 \times 10^{-2}$ & 0.990 \\
\hline 5 & 0.39218469 & 1.65 & $4.042 \times 10^{-2}$ & 0.998 \\
\hline 6 & 0.39918799 & 1.78 & $2.014 \times 10^{-2}$ & 1.005 \\
\hline 7 & 0.40123227 & 1.98 & $1.005 \times 10^{-2}$ & 1.003 \\
\hline 8 & 0.40174937 & 1.92 & $5.022 \times 10^{-3}$ & 1.001 \\
\hline 9 & 0.40188560 & 2.00 & $2.511 \times 10^{-3}$ & 1.000 \\
\hline 10 & 0.40191976 & 2.01 & $1.255 \times 10^{-3}$ & 1.000 \\
\hline 11 & 0.40192822 & 2.00 & - & - \\
\hline 12 & 0.40193032 & - & - & - \\
\hline
\end{tabular}

TABLE 6. Results for the full multigrid algorithm II with $f=1$

\begin{tabular}{|c|c|r|c|c|c|c|}
\hline$k$ & $\kappa_{1, k}$ & $\sigma_{1, k}$ & $\kappa_{2, k}$ & $\sigma_{2, k}$ & $e_{k}$ & $\epsilon_{k}$ \\
\hline 1 & - & - & - & - & $3.938 \times 10^{-1}$ & - \\
\hline 2 & 0.34080586 & - & $-2.0614706 \times 10^{-17}$ & - & $1.829 \times 10^{-1}$ & 1.11 \\
\hline 3 & 0.33610413 & -3.14 & $-1.2218483 \times 10^{-2}$ & 0.32 & $8.644 \times 10^{-2}$ & 1.08 \\
\hline 4 & 0.37747233 & 1.18 & $-2.4317474 \times 10^{-3}$ & 2.47 & $3.634 \times 10^{-2}$ & 1.25 \\
\hline 5 & 0.39567246 & 2.02 & $-6.6497401 \times 10^{-4}$ & 1.83 & $1.054 \times 10^{-2}$ & 1.79 \\
\hline 6 & 0.40014791 & 1.75 & $-1.6711078 \times 10^{-4}$ & 1.99 & $3.307 \times 10^{-3}$ & 1.67 \\
\hline 7 & 0.40147892 & 2.00 & $-4.2006249 \times 10^{-5}$ & 1.99 & $1.028 \times 10^{-3}$ & 1.69 \\
\hline 8 & 0.40181127 & 1.89 & $-1.0519784 \times 10^{-5}$ & 2.00 & $3.384 \times 10^{-4}$ & 1.60 \\
\hline 9 & 0.40190104 & 1.99 & $-2.6327556 \times 10^{-6}$ & 2.00 & $1.140 \times 10^{-4}$ & 1.57 \\
\hline 10 & 0.40192361 & 2.02 & $-6.5851034 \times 10^{-7}$ & 2.00 & $3.923 \times 10^{-5}$ & 1.54 \\
\hline 11 & 0.40192918 & 2.00 & $-1.6466864 \times 10^{-7}$ & 2.00 & - & - \\
\hline 12 & 0.40193057 & - & $-4.1172418 \times 10^{-8}$ & - & - & - \\
\hline
\end{tabular}

In the sixth experiment, we apply the full multigrid algorithm II to (6.7) on the grids depicted in Figure 4. The error $e_{k}$ in the energy norm is defined by $e_{k}=\left|w_{k+1}-I_{k}^{k+1} w_{k}\right|_{H^{1}(\Omega)}$. The convergence rates $\sigma_{1, k}\left(\sigma_{2, k}\right)$ and $\epsilon_{k}$ are computed by (6.8) and (6.6), respectively. The results are tabulated in Table 6.

Experiments 1 and 4 are performed for the sake of comparison with the new algorithms. The convergence rates in the energy norm tend to $2 / 3$ in both experiments. The convergence rates for the stress intensity factors in experiment 4 tend to $1.3 \sim 4 / 3$. In fact, if we extrapolate from Table 6 the value 0.40193103 for the stress intensity factor and compute the convergence rates again, then the last three rates become 1.29, 1.30 and 1.32. The rates for the stress intensity factors in experiment 1 are also decreasing towards $4 / 3$.

Experiments 2 and 5 show the improvement of the full multigrid algorithm I over the standard full multigrid algorithm. The performance of this algorithm is actually better than the prediction of our theory. Note that in both cases the righthand side of the Poisson equation is smooth and hence the regular part $w$ belongs to $H^{(7 / 3)-\epsilon}(\Omega)$ instead of just $H^{2}(\Omega)$. This higher regularity of $w$ and some sort 
TABLE 7. $L^{2}$ convergence of the full multigrid algorithm II with $f=1$

\begin{tabular}{|c|c|c|}
\hline$k$ & $\mu_{k}$ & $\delta_{k}$ \\
\hline 1 & $4.028096 \times 10^{-2}$ & - \\
\hline 2 & $1.420687 \times 10^{-2}$ & 1.50 \\
\hline 3 & $7.475676 \times 10^{-3}$ & 0.93 \\
\hline 4 & $3.121328 \times 10^{-3}$ & 1.26 \\
\hline 5 & $7.693137 \times 10^{-4}$ & 2.02 \\
\hline 6 & $2.272332 \times 10^{-4}$ & 1.76 \\
\hline 7 & $5.608381 \times 10^{-5}$ & 2.02 \\
\hline 8 & $1.499267 \times 10^{-5}$ & 1.90 \\
\hline 9 & $3.770565 \times 10^{-6}$ & 1.99 \\
\hline 10 & $9.353645 \times 10^{-7}$ & 2.01 \\
\hline
\end{tabular}

of superconvergence result are probably responsible for the better than expected performance of this algorithm.

The convergence rates of both of the stress intensity factors tend to 2 in experiments 3 and 6 , as predicted by the theory. The convergence rates in energy norm also tend to 2 in experiment 3, again in agreement with the theory. Note that $\left|s_{1}\right|_{H^{1}(\Omega)} \sim 1.176$ and $\left|s_{2}\right|_{H^{1}(\Omega)} \sim 0.768$, and hence the energy norm error $\left|u-\left(\kappa_{1,11} s_{1}+\kappa_{2,11} s_{2}+w_{11}\right)\right|_{H^{1}(\Omega)}$ in experiment 3 is of order $10^{-7}$, whereas in experiment 1 the energy norm error at level 11 is only of order $10^{-3}$.

The convergence rates in energy norm in experiment 6 do not approach 2 . This may be explained as follows. The order 2 convergence in energy norm depends on a delicate superconvergence result, and the rates computed by (6.6) will tend to the correct order only if there is an asymptotic expansion for the energy norm error instead of just a bound. In experiment 3 , the regular part is actually $C^{\infty}$ and such an asymptotic expansion exists. Hence the order 2 convergence is observed there. On the other hand, the regular part in experiment 6 is only in $H^{3-\epsilon}(\Omega)$ and therefore the asymptotic expansion is absent. Consequently the order 2 convergence in energy norm is not observed.

Note that the estimate (5.10) and the Poincare inequality imply that we have $\mathcal{O}\left(h^{2-\epsilon}\right) L^{2}$-norm convergence for the full multigrid algorithm II. Since the existence of an asymptotic expansion for the $L^{2}$-norm error usually requires less regularity, the $L^{2}$-norm errors in experiment 6 may still show a convergence rate of 2 . We have computed the $L^{2}$-norm errors $\mu_{k}=\left\|w_{k}-w_{k+1}\right\|_{L^{2}(\Omega)}$ and calculated their rates of convergence $\delta_{k}=\log _{2}\left(\mu_{k-1} / \mu_{k}\right)$. The results are displayed in Table 7 . It shows that the rate of convergence in the $L^{2}$-norm is indeed 2 .

\section{CONCluding REMARKS}

The multigrid methods in this paper use the simplest finite element. They can be easily implemented by modifying existing multigrid codes for the P1 finite element. Since the grids are generated by connecting midpoints, it is also easy to parallelize these algorithms. The extension of these methods to domains with cracks is carried out in [17].

For more regular $f$, there exist singular function representations (cf. [27], [24], [23], [39]) where the regular part $w$ is also more regular. In such cases multigrid 
methods with higher orders of convergence can be developed using higher order elements. The generalization of the results of this paper to higher order elements is carried out in [15].

Since the multigrid methods in this paper compute the regular part of the singular solution, superconvergence results (cf. [61], [54], [19], [35]) which require unrealistic regularity on nonsmooth domains become relevant in this approach. Note that other superconvergence results which are less restrictive than the one based on the "uniform band" condition can also be used as they become available.

These methods can also be applied to elasticity problems, transmission problems, problems with mixed Dirichlet and Neumann boundary conditions and fourth order problems. The only prerequisite is the knowledge of the singular function representation, which may not always be readily available. However, in any applications where the stress intensity factors are sought, the singular function representation must be known, as otherwise the concept of stress intensity factors cannot even be defined. We also point out that there exist many algorithms for the computation of the singular functions (cf. [33], [51], [42], [43], [55], [56], [22]).

\section{ACKNOWLEDGMENTS}

The author would like to thank Lars Wahlbin for a discussion of the pollution effect of the corner singularities, Li-yeng Sung for translating some of the Chinese references, and Steve McCormick for many constructive comments.

\section{REFERENCES}

1. I. Babuška, Finite element method for domains with corners, Computing 6 (1970), 264-273. MR 45:2934

2. I. Babuška, R.B. Kellogg and J. Pitkäranta, Direct and inverse error estimates for finite elements with mesh refinements, Numer. Math. 33 (1979), 447-471. MR 81c:65054

3. I. Babuška and A. Miller, The post-processing approach in the finite element method - part 2: The calculation of stress intensity factors, Int. J. Numer. Methods Engrg. 20 (1984), 1111-1129.

4. R.E. Bank and C.C. Douglas, Sharp estimates for multigrid rates of convergence with general smoothing and acceleration, SIAM J. Numer. Anal. 22 (1985), 617-633. MR 87j:65037

5. R.E. Bank and T.F. Dupont, An optimal order process for solving finite element equations, Math. Comp. 36 (1981), 35-51. MR 82b:65113

6. M.Š. Birman and G.E. Skvorcov, On the square summability of the highest derivatives of the solution of the Dirichlet problem in a domain with piecewise smooth boundary, Izv. Vyssh. Uchebn. Zaved. Mat. (1962), no. 5, 12-21 (in Russian). MR 26:2731

7. H. Blum and M. Dobrowolski, On finite element methods for elliptic equations on domains with corners, Computing 28 (1982), 53-63. MR 83a:65108

8. M. Bourlard, M. Dauge, M.-S. Lubuma and S. Nicaise, Coefficients of the singularities for elliptic boundary value problems on domains with conical points III. Finite element methods on polygonal domains, SIAM Numer. Anal. 29 (1992), 136-155. MR 93a:65146

9. D. Braess and W. Hackbusch, A new convergence proof for the multigrid method including the $V$-cycle, SIAM J. Numer. Anal. 20 (1983), 967-975. MR 85h:65233

10. J.H. Bramble, Multigrid Methods, Longman Scientific \& Technical, Essex, 1993. MR 95b:65002

11. J.H. Bramble and S.R. Hilbert, Estimation of linear functionals on Sobolev spaces with applications to Fourier transforms and spline interpolation, SIAM J. Numer. Anal. 7 (1970), 113-124. MR 41:7819

12. J.H. Bramble and J.E. Pasciak, The analysis of smoothers for multigrid algorithms, Math. Comp. 58 (1992), 467-488. MR 92f:65146

13. N New estimates for multilevel algorithms including the V-cycle, Math. Comp. 60 (1993), 447-471. MR 94a:65064 
14. Uniform convergence estimates for multigrid V-cycle algorithms with less than full elliptic regularity, Domain Decomposition Methods in Science and Engineering (Como, 1992), Contemp. Math., vol. 157, Amer. Math. Soc., Providence, RI, 1994, pp. 17-26. MR 95f:65202

15. S.C. Brenner, Overcoming corner singularities by multigrid methods, SIAM J. Numer. Anal (to appear).

16. S.C. Brenner and L.R. Scott, The Mathematical Theory of Finite Element Methods, SpringerVerlag, New York-Berlin-Heidelberg, 1994. MR 95f:65001

17. S.C. Brenner and L.-Y. Sung, Multigrid methods for the computation of singular solutions and stress intensity factors II: crack singularities, BIT 37 (1997), 623-643. CMP 98:05

18. C. Chen, Optimal points of the stresses for triangular linear elements, Numer. Math. J. Chinese Univ. 2 (1980), 12-20 (in Chinese). MR 83d:65279

19. C. Chen and Y. Huang, High Accuracy Theory of Finite Element Methods, Hunan Science and Technology Publishing House, Changsha, 1995 (in Chinese).

20. P.-G. Ciarlet, The Finite Element Method for Elliptic Problems, North Holland, Amsterdam, New York, Oxford, 1978. MR 58:25001

21. P. Clément, Approximation by finite element functions using local regularization, R.A.I.R.O. R-2 (1975), 77-84. MR 53:4569

22. M. Costabel and M. Dauge, Computation of corner singularities in linear elasticity, Boundary Value Problems and Integral equations in Nonsmooth Domains (M. Costabel and M. Dauge, eds.), Lecture Notes in Pure and Appl. Math., 167, Marcel Dekker, New York, 1995, pp. 59-68. MR 95i:65150

23. M. Dauge, Elliptic Boundary Value Problems on Corner Domains, Lecture Notes in Mathematics 1341, Springer-Verlag, Berlin-Heidelberg, 1988. MR 91a:35078

24. M. Dauge, M.-S. Lubuma and S. Nicaise, Coefficient des singularitiés pour le problème de Dirichlet sur un polygone, C. R. Acad. Sci. Paris Sér. I Math. 304 (1987), 483-486. MR 88f:35038

25. M. Dobrowolski, Numerical Approximation of Elliptic Interface and Corner Problems, Habilitationschrift, Bonn, 1981

26. G.H. Golub and C.F. Van Loan, Matrix Computations, Second Edition, The Johns Hopkins University Press, Baltimore, 1989. MR 90d:65055

27. P. Grisvard, Elliptic Problems in Nonsmooth Domains, Pitman, Boston, $1985 . \quad$ MR 86m:35044

28. __ Problèmes aux limites dans les polygones, Mode d'emploi, EDF Bull. Direction Études Rech. Sér. C. Math. Inform. 1 (1986), 21-59. MR 87g:35073

29. __ Singularités en elasticité, Arch. Rat. Mech. Anal. 107 (1989), 157-180. MR 90j:35170

30. Singularities in Boundary Value Problems, Masson, Paris, 1992. MR 93h:35004

31. W. Hackbusch, Multi-Grid Methods and Applications, Springer-Verlag, Berlin, 1985. MR 87e:65082

32. V. Kondratiev, Boundary value problems for elliptic equations in domains with conical or angular points, Tran. Moscow Math. Soc. 16 (1967), 227-313. MR 37:1777

33. D. Leguillon and E. Sanchez-Palencia, Computation of Singular Solutions in Elliptic Problems and Elasticity, Masson, Paris, 1987. MR 90m:73015

34. N. Levine, Superconvergent recovery of the gradient from piecewise linear finite-element approximations, IMA J. Numer. Anal. 5 (1985), 407-427. MR 87b:65206

35. Q. Lin and N. Yan, The Construction and Analysis of Highly Effective Finite Elements, Hebei University Press, Baoding, 1996 (in Chinese).

36. J. Mandel, S. McCormick and R. Bank, Variational multigrid theory, Multigrid Methods (S. McCormick, ed.), Frontiers In Applied Mathematics 3, SIAM, Philadelphia, 1987, pp. 131-177. MR 39m:65004

37. S.F. McCormick, Multigrid methods for variational problems: further results, SIAM J. Numer. Anal. 21 (1984), 255-263. MR 85h:65115

38. M. Mu and H. Huang, Extrapolation acceleration and mg methods for calculating stress intensity factors on re-entrant domains, Math. Numer. Sinica 12 (1990), 54-60 (in Chinese). MR 91e: 73115

39. S.A. Nazarov and B.A. Plamenevsky, Elliptic Problems in Domains with Piecewise Smooth Boundaries, Expositions in Mathematics, vol. 13, de Gruyter, Berlin, New York, 1994. MR 95h:35001 
40. J.A. Nitsche and A.H. Schatz, Interior estimates for Ritz-Galerkin methods, Math. Comp. 28 (1974), 937-958. MR 51:9525

41. L.A. Oganesjan and L.A. Ruhovec, An investigation of the rate of convergence of variationaldifference schemes for second order elliptic equations in a two-dimensional region with smooth boundary, Ž. Vyčisl. Mat. i. Mat. Fiz. 9 (1969), 1102-1120; English transl. in USSR Comput. Math. and Math. Phys. 9 (1969). MR 45:4665

42. P. Papadakis, Computational Aspects of the Determination of the Stress Intensity Factors for Two Dimensional Elasticity, Ph.D. Dissertation, University of Maryland, College Park, 1989.

43. P.J. Papadakis and I. Babuška, A numerical procedure for the determination of certain quantities related to the stress intensity factors in two-dimensional elasticity, Comput. Methods Appl. Mech. Engrg. 122 (1995), 69-92. MR 96a:73016

44. R. Rannacher and R. Scott, Some optimal error estimates for piecewise linear finite element approximations, Math. Comp. 38 (1982), 437-445. MR 83e:65180

45. U. Rüde, Mathematical and Computational Techniques for Multilevel Adaptive Methods, Frontiers in Applied Mathematics 13, SIAM, Philadelphia, 1993. MR 95b:65149

46. A.H. Schatz, An analysis of the finite element method for second order elliptic boundary value problems, Mathematical Theory of Finite and Boundary Element Methods, DMV Seminar Band 15, Birkhäuser, Basel, 1990, pp. 9-133. MR 92f:65004

47. R. Scott, Optimal $L^{\infty}$ estimates for the finite element method on irregular meshes, Math. Comp. 30 (1976), 681-697. MR 55:9560

48. L.R. Scott and S. Zhang, Finite element interpolation of non-smooth functions satisfying boundary conditions, Math. Comp. 54 (1990), 483-493. MR 95j:65021

49. B.A. Szabó and I. Babuška, Computation of the amplitude of stress singular terms for cracks and reentrant corners, Fracture Mechanics: Nineteenth Symposium, ASTM STP 969 (T.A. Cruse, ed.), American Society for Testing and Materials, Philadelphia, 1988, pp. 101-124.

50. H. Triebel, Interpolation Theory, Function Spaces, Differential Operators, North-Holland, Amsterdam, 1978. MR 80i:46032

51. D. Vasilopoulos, On the determination of higher order terms of singular elastic stress fields near corners, Numer. Math. 53 (1988), 51-95. MR 89h:65193

52. L.B. Wahlbin, On the sharpness of certain local estimates for $\stackrel{\circ}{H}^{1}$ projections into finite element spaces: Influence of a reentrant corner, Math. Comp. 42 (1984), 1-8. MR 86b:65129

53. _ L L L L _ behavior in finite element methods, Handbook of Numerical Analysis, Vol.II (P.G.Ciarlet and J.L. Lions, eds.), Finite Element Methods (Part 1), North-Holland, Amsterdam, 1991, pp. 355-522. MR 92f:65001

54. __ Superconvergence in Galerkin Finite Element Methods, Lecture Notes in Mathematics 1605, Springer-Verlag, Berlin, 1995. CMP 97:09

55. Z. Yosibash, Numerical Analysis of Singularities and First Derivatives for Elliptic Boundary Value Problems in Two Dimensions, D.Sc. Dissertation, Sever Institute of Technology, Washington University, St. Louis, 1994.

56. Z. Yosibash and B.A. Szabó, Numerical analysis of singularities in two dimensions, Part 1: Computation of eigenpairs, Int. J. Numer. Methods Engrg. 38 (1996), 2055-2082. MR 96d:73075

57. H. Yserentant, The convergence of multi-level methods for solving finite-element equations in the presence of singularities, Math. Comp. 47 (1986), 399-409. MR 88d:65149

58. S. Zhang, Optimal-order nonnested multigrid methods for solving finite element equations I: On quasi-uniform meshes, Math. Comp. 55 (1990), 23-36. MR 91g:65268

59. _ Optimal-order nonnested multigrid methods for solving finite element equations II: On non-quasi-uniform meshes, Math. Comp. 55 (1990), 439-450. MR 91g:65269

60. __ Optimal-order nonnested multigrid methods for solving finite element equations III: On degenerate meshes, Math. Comp. 64 (1995), 23-49. MR 95c:65194

61. Q.D. Zhu and Q. Lin, The Superconvergence Theory of Finite Elements, Hunan Science and Technology Publishing House, Changsha, 1989 (in Chinese). MR 93j:65191

Department of Mathematics, University of South Carolina, Columbia, SC 29208

E-mail address: brenner@math.sc.edu 